\title{
How light a higgsino or a wino dark matter can become in a compressed scenario of MSSM
}

\author{
Manimala Chakraborti, ${ }^{a}$ Utpal Chattopadhyay ${ }^{b}$ and Sujoy Poddar $^{c}$ \\ ${ }^{a}$ Bethe Center for Theoretical Physics $\&$ Physikalisches Institut der Universität Bonn, \\ Nußallee 12, 53115 Bonn, Germany \\ ${ }^{b}$ Department of Theoretical Physics, Indian Association for the Cultivation of Science, \\ $2 A \&$ B Raja S.C. Mullick Road, Jadavpur, Kolkata 700 032, India \\ ${ }^{c}$ Department of Physics, Netaji Nagar Day College, \\ 170/436, N.S.C. Bose Road, Kolkata - 700092, India \\ E-mail: mani.chakraborti@gmail.com, tpuc@iacs.res.in, \\ sujoy ·phy@gmail.com
}

ABSTRACT: Higgsinos and Wino have strong motivations for being Dark Matter (DM) candidates in supersymmetry, but their annihilation cross sections are quite large. For thermal generation and a single component DM setup the higgsinos or wino may have masses of around 1 or $2-3 \mathrm{TeV}$ respectively. For such DM candidates, a small amount of slepton coannihilation may decrease the effective DM annihilation cross section. This, in turn reduces the lower limit of the relic density satisfied DM mass by more than $50 \%$. Almost a similar degree of reduction of the same limit is also seen for squark coannihilations. However, on the contrary, for near degeneracy of squarks and higgsino DM, near its generic upper limit, the associated coannihilations may decrease the relic density, thus extending the upper limit towards higher DM masses. We also compute the direct and indirect detection signals. Here, because of the quasi-mass degeneracy of the squarks and the LSP, we come across a situation where squark exchange diagrams may contribute significantly or more strongly than the Higgs exchange contributions in the spin-independent direct detection cross section of DM. For the higgsino-DM scenario, we observe that a DM mass of $600 \mathrm{GeV}$ to be consistent with WMAP/PLANCK and LUX data for sfermion coannihilations. The LUX data itself excludes the region of 450 to $600 \mathrm{GeV}$, by a half order of magnitude of the cross-section, well below the associated uncertainty. The similar combined lower limit for a wino DM is about $1.1 \mathrm{TeV}$. There is hardly any collider bound from the LHC for squarks and sleptons in such a compressed scenario where sfermion masses are close to the mass of a higgsino/wino LSP.

KEYwORDS: Supersymmetry Phenomenology

ARXIV EPRINT: 1702.03954 


\section{Contents}

1 Introduction 1

2 Sfermion coannihilations with Higgsino and wino types of LSP 5

3 Results for relic density $\quad 7$

$\begin{array}{lll}3.1 & \text { Quasi degeneracy of electroweakino masses } & 7\end{array}$

3.2 Higgsino dominated LSP 8

$\begin{array}{ll}3.3 & \text { Wino dominated LSP } \\ \end{array}$

4 Direct and indirect detection of DM $\quad \mathbf{1 5}$

$\begin{array}{lll}4.1 & \text { Direct detection } & 15\end{array}$

4.2 Indirect detection 20

5 Conclusion $\quad 25$

\section{Introduction}

One of the most interesting features of low energy supersymmetry (SUSY) [1-8] is that it can provide with a viable candidate for dark matter (DM) $[9,10]$. The lightest supersymmetric particle (LSP), typically the lightest neutralino, in R-parity conserving scenarios of the Minimal Supersymmetric Standard Model (MSSM) [1-8] is a strong candidate for DM. In MSSM, the neutralinos are composed of gauginos (bino and wino) and higgsinos. Whether the LSP in a particular MSSM scenario is able to satisfy the right relic abundance, depends crucially on its gaugino-higgsino compositions. For example, in the minimal supergravity model (mSUGRA) [7, 8, 11-16] that assumes SUSY breaking at a high scale, the LSP is bino-like for most of the parameter space. Bino being a gauge singlet, its annihilation occurs mainly through t-channel exchange of sfermions, giving rise to the bulk annihilation region [4-6]. With the discovery of a $125 \mathrm{GeV}$ Higgs boson at the Large Hadron Collider (LHC) of CERN [17-19], the sfermion masses are pushed towards higher values, particularly for the universal models like mSUGRA. This makes bulk annihilation rather an inefficient mechanism for obtaining the right relic density [20-25]. It is however possible to have a bino-LSP giving the correct abundance of DM in some specific regions of parameter space of mSUGRA, like the stau coannihilation, funnel (resonant Higgs annihilation) or hyperbolic branch (HB) [26-28]/ focus point (FP) [29-35] regions. While most of the former two regions of parameter space are ruled out by the Higgs data [17-19], a large part of the HB/FP region, which corresponds to a significant degree of bino-higgsino mixing, is disfavored by DM direct detection experiments [36-39]. There is also a higgsino LSP region in mSUGRA but this comes with a baggage of a very large gluino mass and a 
heavy SUSY spectra in general. A SUSY scenario where the spectra may not necessarily be so heavy while the LSP is still highly higgsino dominated in nature may be realized in a few nonuniversal gaugino mass models [40-67], nonuniversal Higgs scalar models [68-70] or models with non-holomorphic soft terms [71, 72].

Apart from bino, one may have the right relic abundance in MSSM if the LSP is a higgsino, wino or a well-managed admixture of bino, wino and higgsinos [73]. For the higgsino-like LSP scenario, the lightest neutralino $\left(\widetilde{\chi}_{1}^{0}\right)$ mass is close to the value of the higgsino mixing parameter $\mu$. In this case the lightest chargino $\widetilde{\chi}_{1}^{ \pm}$and the second lightest neutralino $\widetilde{\chi}_{2}^{0}$ are almost mass degenerate with $\widetilde{\chi}_{1}^{0}$ with their masses are close to $\mu$. For a thermally generated single component dark matter, it has been typically accepted that the LSP mass for obtaining the right $\mathrm{DM}$ abundance is around $1 \mathrm{TeV}$ for a higgsino-like LSP $[27,28,36-39]$. Below this limit the annihilation and coannihilations among $\widetilde{\chi}_{1}^{0}, \widetilde{\chi}_{2}^{0}$ and $\tilde{\chi}_{1}^{ \pm}$are too strong causing the DM relic density to become underabundant.

On the other hand, a wino-like LSP may be possible to realize if $M_{2}<\left(M_{1}, \mu\right)$ where $M_{2}$ and $M_{1}$ are the $\mathrm{SU}(2)_{L}$ and $\mathrm{U}(1)_{Y}$ gaugino mass parameters respectively. A wino may be the candidate for LSP in many theoretically well-motivated models like the Anomaly Mediated Supersymmetry Breaking (AMSB) [74-77]. For a wino LSP, $m_{\widetilde{\chi}_{1}^{0}}$ and $m_{\widetilde{\chi}_{1}^{ \pm}}$lie very close to $M_{2}$ allowing strong coannihilations. A thermally generated wino dark matter is underabundant up to $\sim 2 \mathrm{TeV}$. It satisfies the relic density data for a wino mass of $2-3 \mathrm{TeV}^{1}[78-88]$.

Focusing on the collider information as received from the LHC we note that the SUSY searches at the LHC in generic MSSM spectra i.e. in an uncompressed sparticle mass scenario, have imposed stringent bounds on the masses of strong sector sparticles [89100]. The strong sector scalar masses are increasingly pushed above a $\mathrm{TeV}$ regime that is even superseded by the gluino mass limits. On the other hand, the direct mass bounds on the electroweak (EW) sector sparticles from the LHC searches are rather mild [101111]. In the context of mass limits of sparticles, we must however remember that the LHC searches are restricted to the so-called "simplified models" that are characterized by certain assumptions on sparticle masses and compositions of the EW sector gauginos (electroweakinos). The searches in the $3 l+\mathbb{F}_{T}$ channel [112] for example consider $\widetilde{\chi}_{1}^{0}$ to be purely a bino and $\widetilde{\chi}_{1}^{ \pm} / \widetilde{\chi}_{2}^{0}$ to be completely wino dominated. Imposing basic constraints like the Higgs mass, dark matter relic density and muon $g-2$ there have been studies that effectively probed the SUSY parameter space for the above types of electroweakinos [113132]. However, it turns out that the collider limits get significantly degraded once we start varying the composition of the electroweakinos. This may be seen in ref. [133] where the authors considered $\widetilde{\chi}_{1}^{ \pm}$to be higgsino dominated or a mixture of a wino and higgsinos in a bino dominated LSP $\left(\widetilde{\chi}_{1}^{0}\right)$ situation. Similar to the above, changed composition of the LSP itself may significantly alter the collider limits. For example, the trilepton search limits are hardly of any importance in a higgsino dominated LSP scenario where $\widetilde{\chi}_{1}^{0}, \widetilde{\chi}_{2}^{0}$ and $\widetilde{\chi}_{1}^{ \pm}$are almost mass degenerate. This is simply because the resulting leptons come out to be very

\footnotetext{
${ }^{1}$ The spread of results depends on purity of wino, the extent of decoupling of the squark masses as well as the higgsino mass parameter $\mu$, inclusion of non-perturbative effects like Sommerfeld corrections etc. We will come back to it in section 3.3.
} 
soft. For collider studies of benchmark points that satisfy the observed relic density range, one may however use monojet $+\mathbb{E}_{T}$ analysis $[134,135]$. However, the bounds are seen to be very weak [136]. Apart from all the above, collider bounds of sfermions including also squarks get severely diluted if one considers a compressed scenario of sparticle masses where the LSP is higgsino/wino dominated in its composition with its mass close to that of sleptons and/or squarks as appropriate in a LSP-sfermion coannihilation study.

In this analysis we use a compressed SUSY scenario in a phenomenological MSSM (pMSSM) [137] framework so as to explore how light the higgsinos and wino can become while having relic density values within the phenomenologically accepted range. We will consider appropriate coannihilations of the LSP separately with sleptons and squarks or both of them together.

We will now briefly outline the commonly explored coannihilation scenarios. To determine the relic density including coannihilations one computes a thermally averaged effective annihilation cross-section $\left\langle\sigma_{\text {eff }} v\right\rangle$ for the LSP [138]. $\left\langle\sigma_{\text {eff }} v\right\rangle$ is obtained from self-annihilation and various coannihilation cross-sections that are weighted by factors exponentially suppressed by relative mass differences between the DM and the coannihilating partners. The DM relic density is inversely proportional to $\left\langle\sigma_{\text {eff }} v\right\rangle$. In the context of mSUGRA, excluding a few specific regions of parameter space, the LSP is generally bino $(\tilde{B})$-dominated in its composition $[7,8]$. Except the t-channel slepton exchange that requires very light sleptons or the s-channel Higgs modes, a bino typically undergoes a tiny amount of self-annihilation that leads to overabundance in most of the mSUGRA parameter space. The situation changes when bino-slepton or in particular bino-stau coannihilation comes into the picture. Here the non-relativistic threshold S-wave coannihilation cross-sections such as $\sigma_{\tilde{B}-l_{R}}$ and $\sigma_{l_{R}-l_{R}^{*}}, l_{R}$ being the right handed stau, are not suppressed by fermionic mass factors. The above coannihilation cross-sections are much larger than the self-annihilation cross-section of a highly bino-dominated LSP [139]. Thus, in mSUGRA, the coannihilation of $\widetilde{\chi}_{1}^{0}$ with $\tilde{\tau}_{1}$ is able to reduce the relic density to fall within the WMAP/PLANCK [140, 141] specified range. A detailed analysis was made in ref. [142] where all possible kinds of coannihilations were considered in a binodominated LSP scenario in an mSUGRA setup. However, we must remember that masses of sparticles in mSUGRA are correlated that causes mostly LSP-stau coannihilations to be relevant. Thus, a significant amount of change in the DM relic density via coannihilations, leads to an acceptable value, but this is possible only in a limited zone of parameter space.

Models with essentially unconstrained sparticle masses such as pMSSM when considered in a compressed scenario are able to probe the true potential of coannihilations. Here, the LSP may find several coannihilating partners almost mass-degenerate with itself that would overcome the associated exponential suppression encountered in computing the effective annihilation cross section. In this analysis, we will focus on higgsino and wino dominated LSPs that undergo coannihilations with sfermions, both sleptons and squarks, separately or together. We will see that there may be rather uncommon occurrences when coannihilations may potentially cause a decrease in the effective annihilation cross-sections, thereby causing an increase in the DM relic density. This was discussed in refs. [143, 144] where the latter reference named the coannihilating species as parasite degree of freedom 
in which the authors investigated the role of sleptons coannihilating with the LSP. In this work, we will systematically analyze the effects of coannihilations with all the sfermions in a compressed scenario and probe the mass reach of the LSP as a higgsino/wino in relation to the latest phenomenological constraints involving dark matter, Higgs mass and the relevant bounds from collider data.

We emphasize that the effect of considering a coannihilating particle, in particular whether it would cause a decrease or increase of the relic density depends on several factors $[138,143]$, namely, i) the annihilation cross section $\sigma_{\chi_{i}^{\prime}-\chi_{0}}$ of the coannihilating particle $\chi_{i}^{\prime}$ with the LSP $\left(\chi_{0}\right)$, ii) the cross section $\sigma_{\chi_{i}^{\prime}-\chi_{i}^{\prime}}$ for the coannihilating particle annihilating with itself, iii) $\sigma_{\chi_{i}^{\prime}-\chi_{j}^{\prime}}$, where $i$ and $j$ refer to different species of coannihilating particles, iv) the relative mass gap between the sparticles namely, $\delta_{i}=\frac{m_{\chi_{i}^{\prime}}-m_{\chi_{0}}}{m_{\chi_{0}}}$ or $\delta_{i j}=\frac{m_{\chi_{i}^{\prime}}-m_{\chi_{j}}}{m_{\chi_{i}}}$, thereby on the extent of exponential suppression, v) appropriate weight factors arising out of the degrees of freedom of the associated particles undergoing coannihilations.

We must remember that while a bino does not have any gauge charge, a higgsino (wino) is associated with isospin $\frac{1}{2}(1)$. This translates into a larger internal degrees of freedom, namely 8 for a higgsino and 6 for a wino type of LSP considering its Majorana nature. Expectedly, a large number of internal degrees of freedom contributes more toward the self-annihilation cross section of the LSP. In addition to the above, one must also consider large coannihilations involving candidates like $\widetilde{\chi}_{1}^{ \pm}, \widetilde{\chi}_{2}^{0}$ for a higgsino type of LSP and $\widetilde{\chi}_{1}^{ \pm}$for a wino type of the same. All the above lead to a substantially large effective annihilation cross-section for the above two types of LSP. Among the coannihilating sfermions, the left handed ones have larger internal degrees of freedom. This further gets multiplied by the color degrees of freedom for squarks. In computing the effective annihilation rates out of individual cross-sections, one notes that the associated weight factors for different coannihilating species play very important roles in either decreasing or increasing the total rate itself. As mentioned before, unlike a bino LSP, a higgsino or a wino LSP is intrinsically associated with a larger amount of self-annihilation as well as LSP-electroweakino coannihilations. We will refer this as a generic higgsino or wino DM scenario. It turns out that in a compressed sfermion scenario all the appropriate degrees of freedom of the coannihilating sfermions may contribute to the averaging process toward $\left\langle\sigma_{\text {eff }} v\right\rangle$ in such a way that the latter becomes smaller than the case of having no sfermion coannihilations over most of the parameter space satisfying the DM relic density limits. Thus, for a given LSP mass the relic density increases. This on the other hand is synonymous with a decreased lower limit of the mass of LSP satisfying the WMAP/PLANCK data. However, apart from the typical trend mentioned above, we will come across a parameter region corresponding to a higgsino as LSP where the outcome due to squark-squark coannihilations may become dominant over the electroweakino part of the LSP depletion cross section.

We will also study the direct and indirect detection prospects for the types of DM considered in this analysis. The LUX [145] experiment puts strong bounds on spin-independent (SI) DM direct detection cross-sections. The $\widetilde{\chi}_{1}^{0}$-nucleon scattering cross-section that LUX relies on is enhanced for sufficient gaugino-higgsino mixing [146]. However, in our scenario the cross section is supposed to be small for LSP being so pure, either a higgsino or a 
wino. On the other hand, the DM indirect detection experiments $[147,148]$ look for signals coming from stable final state particles of DM annihilation processes in the solar or galactic cores. Since the sfermion-coannihilations make the smaller DM mass zones to become valid in relation to the relic density data, it is important to find whether the indirect detection rates can also be large for much smaller values of higgsino or wino masses satisfying the DM relic density limits.

The paper is organized as follows. In section 2 we briefly discuss the effect of sfermion coannihilations in the calculation of the effective cross-section. In section 3 we present the relic density computation results for higgsinos and wino types of LSP by considering slepton and squark coannihilations separately or together. We will start the section by discussing the roles of the relevant electroweakino mass differences that potentially affect the higgsino and wino relic density results. Constraints coming from the direct and indirect DM detection experiments on our results are discussed in section 4. Finally, we conclude in section 5 .

\section{Sfermion coannihilations with Higgsino and wino types of LSP}

Let us consider the evolution of a class of particles $\chi_{i}, i=1, \ldots N$, in the Early Universe. We assume the particles are different from SM candidates by assuming an R-parity conserved scenario of SUSY. The Boltzmann equation governing the number density $n_{i}$ of the i-th kind of particle at any instant of time $t$ is given by [143],

$$
\begin{aligned}
\frac{d n_{i}}{d t}= & -3 H n_{i}-\sum_{j=1}^{N}\left\langle\sigma_{i j} v_{i j}\right\rangle\left(n_{i} n_{j}-n_{i}^{\mathrm{eq}} n_{j}^{\mathrm{eq}}\right) \\
& -\sum_{j \neq i}\left[\left\langle\sigma_{X i j}^{\prime} v_{i j}\right\rangle\left(n_{i} n_{X}-n_{i}^{\mathrm{eq}} n_{X}^{\mathrm{eq}}\right)-\left\langle\sigma_{X j i}^{\prime} v_{i j}\right\rangle\left(n_{j} n_{X}-n_{j}^{\mathrm{eq}} n_{X}^{\mathrm{eq}}\right)\right] \\
& -\sum_{j \neq i}\left[\Gamma_{i j}\left(n_{i}-n_{i}^{\mathrm{eq}}\right)-\Gamma_{j i}\left(n_{j}-n_{j}^{\mathrm{eq}}\right)\right]
\end{aligned}
$$

where the first term is due to the expansion of the universe and $H$ is the Hubble parameter [10]. The second term arises because of coannihilations between $i$-th and $j$-th sparticles leading to SM particles in the final state i.e. for processes like $\chi_{i} \chi_{j} \rightarrow X$. The total crosssection for scattering off the cosmic thermal background, $\chi_{i} X \rightarrow \chi_{j} Y$ is given by $\sigma_{X i j}^{\prime}$, where $\mathrm{X}$ and $\mathrm{Y}$ are SM type of particles. The last term describes the decay of $\chi_{i}$ and $\Gamma_{i j}$ refers to the total decay width for the processes $\chi_{i} \rightarrow \chi_{j} X$. Since in an R-parity conserving scenario all the existing sparticles will eventually decay into the LSP, its number density is given as $n=\sum_{i=1}^{N} n_{i}$. Now, an assumption for the distribution of $\widetilde{\chi}_{1}^{0}$ maintaining its thermal equilibrium value i.e. $\frac{n_{i}}{n} \simeq \frac{n_{i}^{\text {eq }}}{n^{\text {eq }}}$, eq. (2.1) leads to,

$$
\frac{d n}{d t}=-3 H n-\left\langle\sigma_{\mathrm{eff}} v\right\rangle\left(n^{2}-n_{\mathrm{eq}}^{2}\right)
$$

where,

$$
\left\langle\sigma_{\mathrm{eff}} v\right\rangle=\frac{\sum_{i j}\left\langle\sigma_{i j} v_{i j}\right\rangle n_{i}^{\mathrm{eq}} n_{j}^{\mathrm{eq}}}{n^{\mathrm{eq}}{ }^{2}}
$$


In the non-relativistic approximation, one has,

$$
\frac{n_{i}^{e q}}{n^{e q}}=\frac{g_{i} \exp \left(-x \delta_{i}\right)\left(1+\delta_{i}\right)^{3 / 2}}{g_{\mathrm{eff}}},
$$

where $g_{i}$ is the number of internal degrees of freedom of the $i$-th particle, $\delta_{i}=\frac{m_{i}-m_{1}}{m_{1}}$ (for $i>1), x=\frac{m_{1}}{T}, m_{1}$ being the mass of the LSP and $g_{\text {eff }}=\sum_{i=1}^{N} g_{i} \exp \left(-x \delta_{i}\right)\left(1+\delta_{i}\right)^{3 / 2}$.

In this analysis with slepton and squark coannihilations each $\delta_{i}$ corresponding to a sfermion type $i$ is allowed to vary up to a chosen limit $\delta_{\max }$. Thus, each sfermion mass $m_{\tilde{f}_{i}}$ will have an upper limit of $m_{\tilde{\chi}_{1}^{0}}\left(1+\delta_{\max }\right) . \delta_{\max }$ is chosen as $20 \%$ keeping in mind the exponential suppression within eq. (2.4). Considering a slepton coannihilation scenario in pMSSM, as an example, we note that the first two generations of sleptons do not differ much in their mass values among themselves and these will be close to $M_{\tilde{l}}\left(=M_{\tilde{l}_{R}}\right)$, the pMSSM common slepton mass parameter. ${ }^{2}$ First, let us consider only the sleptons of the first two generations to undergo coannihilations. For a given $m_{\widetilde{\chi}_{1}^{0}}$ and $\delta_{\max }$, the sleptons will have a maximum mass value of $m_{\widetilde{\chi}_{1}^{0}}\left(1+\delta_{\max }\right)$ and this will be close to the maximum value of $M_{\tilde{l}}$. For a given LSP mass, calling the average of all the associated $\delta_{i}$ values as $\delta$, one finds that the highest possible $\delta$ becomes close to $\delta_{\max }$, the chosen degree of maximum relative mass deviation, irrespective of $\tan \beta$. Next, we allow the third generation of sleptons to have mass values in the coannihilation zone. Consequent of the L-R mixing effect (which is more prominent for a larger $\tan \beta), m_{\tilde{\tau}_{1}}$ and $m_{\tilde{\tau}_{2}}$ are largely separated among themselves. Only the heavier stau will have its maximum mass value near $m_{\widetilde{\chi}_{1}^{0}}\left(1+\delta_{\max }\right)$ and all other sleptons will have much smaller masses. Thus, the reach of $M_{\tilde{l}}$ becomes smaller and so is the average value of all the three generations of slepton masses. Hence, the average relative deviation $\delta$ will have its maximum value significantly smaller than $\delta_{\max }$, an effect that would increase with $\tan \beta$. Additionally, when LSP mass is in the smaller zone meaning a stronger degree of electroweakino coannihilations, the requirement of slepton coannihilations increases so as the satisfy the relic density limits. Thus, the slepton masses are needed to stay within the close vicinity of $m_{\widetilde{\chi}_{1}^{0}}$. Consequently, $\delta$ as well as its range of variation both become smaller for a given mass of the LSP. Here, the aforesaid range becomes smaller because of the stau L-R mixing since coannihilation effects of both the staus are quite required in the process. In other words, the stau masses cannot be too far away from $m_{\widetilde{\chi}_{1}^{0}}$, or the associated $\delta_{i}$ values can not be large, thus avoiding an exponential suppression. It follows that the minimum mass of the LSP satisfying eq. (3.1) becomes larger for a larger $\tan \beta$. Similarly, squark coannihilations, in principle, will also show qualitatively identical behaviour ${ }^{3}$ based on the availability of the all the generation of squarks for coannihilations. However, we will consider only the first two generations of squarks in this study keeping the top-squarks in a decoupled zone because of a very large L-R mixing, particularly arising from the requirement of satisfying the Higgs mass data.

In regard to a book-keeping of the internal degrees of freedom (d.o.f.) we note that for a given generation of the right and left handed sleptons like $\tilde{\tau}_{R, L}$ there are 2 internal d.o.f.

\footnotetext{
${ }^{2}$ Only small differences come from the D-term contributions of the left and the right sleptons as well as sneutrinos.

${ }^{3}$ We will however point out a difference for the higgsino LSP case in the heavier limit of the LSP.
} 
available for each $\mathrm{R}$ and $\mathrm{L}$ while the sneutrino $\tilde{\nu}_{\tau}$ along with its anti-particle would have one internal d.o.f. each. Thus, for a compressed slepton spectra undergoing coannihilations with the LSP the total number of internal d.o.f. for all the three generations of sleptons would be 18. For the squark coannihilations with only two generations are considered in the analysis, the resulting internal d.o.f. amounts to 48 after accounting for the color d.o.f..

\section{Results for relic density}

In spite of the fact that the PLANCK [141] data for the DM relic density has a very small uncertainty $\left(\Omega_{C D M} h^{2}=0.1199 \pm 0.0022\right)$, we note that there is about a $10 \%$ level of theoretical uncertainty in computing the SUSY DM relic density [149, 150]. This is approximately six times the observational uncertainty, as concluded in refs. $[149,150]$. It was shown that higher order SUSY-QCD corrections may cause a significant degree of shift of the relic density in some scenarios and the uncertainty arising out of renormalization scheme and scale variations can be quite significant. Several recent analyses used such a degree of theoretical error or even more (see for example refs. [151, 152]). Thus, we will use $\Omega_{\widetilde{\chi}_{1}^{0}} h^{2}=0.12 \pm 0.012$ that leads to the following bounds.

$$
0.108<\Omega_{\widetilde{\chi}_{1}^{0}} h^{2}<0.132 .
$$

We use the code SuSpect (version 2.43) [153] for spectrum generation. For the calculation of DM relic density and direct and indirect detection observables we use the code micrOMEGAs version 3.2 [154]. We have also verified agreement with the code DarkSUSY [155] by choosing various representative points over the parameter space.

Throughout the analysis we impose the Higgs mass range of 122 to $128 \mathrm{GeV}$ considering the uncertainty in computing SUSY Higgs mass $m_{h}$ with radiative corrections [156-161].

\subsection{Quasi degeneracy of electroweakino masses}

Since the electroweakino coannihilations play a dominant role in computing the DM relic density both for higgsino and wino types of LSPs, it is important to discuss briefly the role of the appropriate electroweakino mass differences [162-168]. In regard to a higgsino type of LSP, both the mass differences $m_{\widetilde{\chi}_{1}^{ \pm}}-m_{\widetilde{\chi}_{1}^{0}}$ and $m_{\widetilde{\chi}_{2}^{0}}-m_{\widetilde{\chi}_{1}^{0}}$ are important. Typically the latter is about double the former at the tree level [164]. For a higgsino type of LSP, with $M_{2}>\mu, M_{W}$, an expansion in $1 / M_{2}$ leads to the following tree level mass difference [166].

$$
m_{\widetilde{\chi}_{1}^{ \pm}}-m_{\widetilde{\chi}_{1}^{0}}=\left[\frac{M_{2}}{M_{1}} \tan ^{2} \theta_{W}+1+\operatorname{sgn} \mu\left(\frac{M_{2}}{M_{1}} \tan ^{2} \theta_{W}-1\right) \sin 2 \beta\right] \frac{M_{W}^{2}}{2 M_{2}}+\mathcal{O}\left(\frac{1}{M_{2}^{2}}\right) .
$$

In the wino limit of the LSP, one has $M_{2}<|\mu|,\left|M_{1}\right|$ causing the difference $m_{\widetilde{\chi}_{1}^{ \pm}}-m_{\widetilde{\chi}_{1}^{0}}$ to become small. An expansion in $1 / \mu$ leads to the following tree level relationship [166].

$$
\begin{aligned}
m_{\widetilde{\chi}_{1}^{ \pm}}-m_{\widetilde{\chi}_{1}^{0}}= & \frac{M_{W}^{2}}{\mu^{2}} \frac{M_{W}^{2}}{M_{1}-M_{2}} \tan ^{2} \theta_{W} \sin ^{2} 2 \beta+2 \frac{M_{W}^{4} M_{2} \sin 2 \beta}{\left(M_{1}-M_{2}\right) \mu^{3}} \tan ^{2} \theta_{W} \\
& +\frac{M_{W}^{6} \sin ^{3} 2 \beta}{\left(M_{1}-M_{2}\right)^{2} \mu^{3}} \tan ^{2} \theta_{W}\left(\tan ^{2} \theta_{W}-1\right)+\mathcal{O}\left(\frac{1}{\mu^{4}}\right) .
\end{aligned}
$$


Additional suppression comes for large $\tan \beta$ since $\sin ^{2}(2 \beta) \sim 4 / \tan ^{2} \beta$. Thus, the terms up to the order $1 / \mu^{3}$ are suppressed indicating the lowest contributing order to be $1 / \mu^{4}$ which is given as follows [167].

$$
m_{\widetilde{\chi}_{1}^{ \pm}}-m_{\widetilde{\chi}_{1}^{0}}=\frac{M_{2} M_{W}^{4}}{2 \mu^{4}}\left(1+\frac{2 M_{2} \tan ^{2} \theta_{W}}{M_{1}-M_{2}}\right)+\mathcal{O}\left(\frac{1}{\mu^{6}}\right), \quad \text { for } \tan \beta \rightarrow \infty .
$$

One finds that the above mass splitting is small even for moderate values of $\mu$. Thus, the radiative corrections for the two electroweakino masses or rather that of their difference become important [169-173]. The dominant corrections to the masses come from top-stop and $\gamma(Z)$-higgsino loops $[169,170]$. On the other hand, the renormalization of the mass difference is controlled by the gauge boson loops as pointed out in refs. [165, 168]. However, in our analysis we find a non-negligible reduction in the mass gap when $\mu$ and/or squark masses are taken to be very large $(\sim 10 \mathrm{TeV})$ and we agree with the conclusion of ref. [164] in this regard. In this analysis, since we are looking for slepton and squark coannihilations with the LSP while trying to probe the lower mass limit of the latter, we undertake a minimalistic approach of considering not too much different mass values for the scalars among themselves. We also limit $\mu$ so as to have only an adequate degree of wino purity of the LSP while trying to respect naturalness [26-35, 174-176] as far as possible. Hence, we will prefer not to take very large values for the scalar masses or $\mu$.

\subsection{Higgsino dominated LSP}

A higgsino dominated LSP with mass close to $\mu$ can be made out of the choice $M_{2}>M_{1}>$ $\mu$. We will quantify the degree of higgsino content of the LSP via $Z_{h}$ defined as $Z_{h}=$ $\left(N_{13}^{2}+N_{14}^{2}\right)$, where $N_{i j}, i, j=1,2,3,4$ are the elements of the neutralino-mass diagonalizing matrix [7, 8]. In particular, we have used the following choice: $M_{1}=2 \mu$ and $M_{2}=2.4 \mu$. We then vary $\mu$ in the range of $100 \mathrm{GeV}<\mu<2 \mathrm{TeV}$ that covers the typical relic density satisfied higgsino mass zone of $1 \mathrm{TeV}$. In a correlated scanning setup, for each value of $\mu$ we vary the common slepton mass parameter for all the three generations of sleptons within the range $50 \%$ below and above the value of $\mu$. The common squark mass parameters are chosen to be large $(3 \mathrm{TeV})$. For squarks, we allow coannihilations only with the first two generations for reasons described in section 2 while taking sleptons as well as the third generation of squarks to be heavy $(3 \mathrm{TeV})$. As before, we vary $\mu$ in the range of $100 \mathrm{GeV}$ $<\mu<2 \mathrm{TeV}$. Then, for each value of $\mu$ we vary the common squark mass parameters for the first two generations within the range $50 \%$ below and above the value of $\mu$.

Furthermore, while $\mu$ being varied, we scan the trilinear soft breaking parameter $A_{t}$ from $-2 \mathrm{TeV}$ to $-7 \mathrm{TeV}$, so as to satisfy the higgs mass data. However, we must emphasize that the choice of $A_{t}$ has a very small impact in our study of sfermion coannihilations. The squark mass parameters of the third generation as well as the electroweak symmetry breaking (EWSB) scale are taken as $3 \mathrm{TeV}$ throughout the slepton and squark coannihilation studies. The $\mathrm{SU}(3)_{C}$ gaugino mass parameter $M_{3}$ is also chosen to be $3 \mathrm{TeV}$ whereas the mass of the CP-odd Higgs $\left(M_{A}\right)$ is set at $5 \mathrm{TeV}$. The latter avoids a Higgs resonance annihilation region. In order to study the effect of slepton coannihilations on the relic density of DM, we make sure that the physical slepton masses stay within $20 \%$ of the LSP 


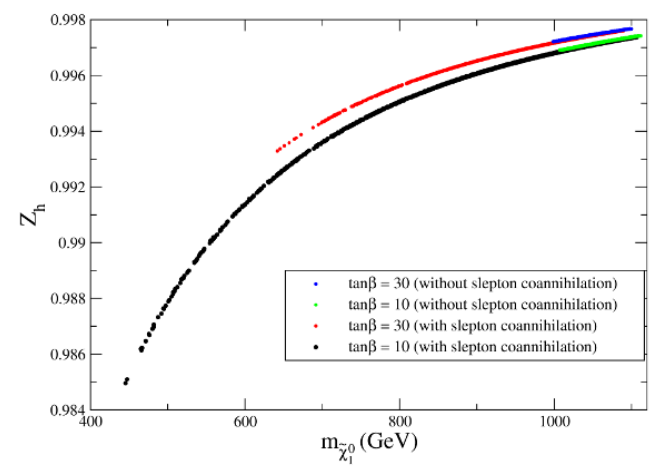

(a)

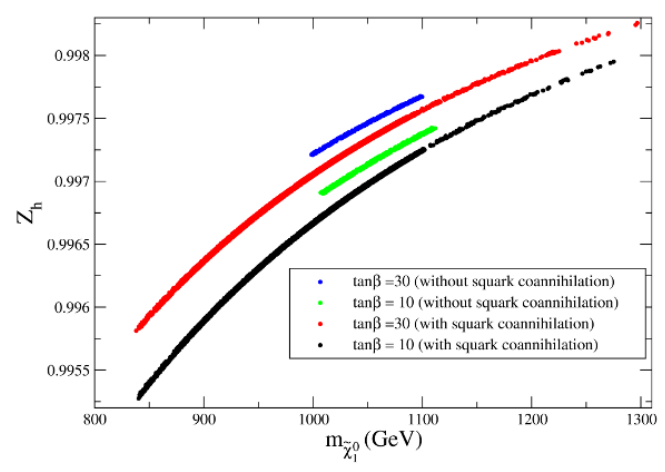

(b)

Figure 1. a) Plot of higgsino fraction $Z_{h}$ vs LSP mass for a higgsino dominated LSP when the LSP coannihilates with sleptons and sneutrinos of all the three generations apart from the usual LSP- $\widetilde{\chi}_{1}^{ \pm}$ and LSP- $\widetilde{\chi}_{2}^{0}$ coannihilations for $\tan \beta=10$ and 30 as appropriate to the case of a higgsino-type of LSP. The plot is obtained by varying $\mu$ in a correlated higgsino-gaugino mass setup as explained in the text. The black and red points refer to $\tan \beta=10$ and 30 respectively. The reference results with no slepton coannihilations are shown in green and blue points for $\tan \beta=10$ and 30 respectively. All the points in the plot satisfy the lower and upper limits of DM relic density (eq. (3.1)). Clearly, the above sfermion coannihilations reduce the lower limit of the LSP mass satisfying eq. (3.1) to $m_{\widetilde{\chi}_{1}^{0}} \simeq 450 \mathrm{GeV}$ from about $1 \mathrm{TeV}$. b) Similar plot with LSP-squark coannihilations along with reference cases where squarks refer only to the first two generations. The color codes are same as those of (a). Reduction of the lower limit and enhancement of the upper limit of the LSP mass are notable.

mass irrespective of the generation. The same is true for the case of first two generations of squarks while we analyze the effects of squark coannihilations. We like to emphasize that with the above nearly degenerate squark masses close to that of the LSP, the commonly discussed LHC limits [177] for squarks would not apply to our scenario.

Figure 1(a) shows the scatter plot of the higgsino fraction $Z_{h}$ vs LSP mass when the LSP efficiently coannihilates with sleptons and sneutrinos of all the three generations. We note that the LSP-sfermion coannihilations take place in the background of strong electroweakino coannihilations. The significance of higgsino purity level in turn is directly related to the level of coannihilations between the electroweakino states $\widetilde{\chi}_{1}^{0}, \widetilde{\chi}_{2}^{0}$ and $\widetilde{\chi}_{1}^{ \pm}$. The squarks are taken to be very heavy $(3 \mathrm{TeV})$. The reference results for the generic higgsino LSP or the case of no sfermion coannihilations are shown in green and blue points for $\tan \beta=10$ and 30 respectively. The black and red points refer to $\tan \beta=10$ and 30 respectively for the cases with slepton coannihilations. All the points in the scatter plot satisfy the lower and upper limits of DM relic density. Clearly as seen in figure 1(a), the slepton coannihilations reduce the lower limit of the LSP mass satisfying eq. (3.1) to $m_{\widetilde{\chi}_{1}^{0}} \simeq 450 \mathrm{GeV}$ for $\tan \beta=10$ and $\sim 640 \mathrm{GeV}$ for $\tan \beta=30$ respectively from about $1 \mathrm{TeV}$ corresponding to the generic higgsino DM result. There is hardly any change in the upper limit of the mass of LSP in this regard. The difference of the lower limits for the two values of $\tan \beta$ for the case of slepton coannihilations arises from the L-R mixing of 


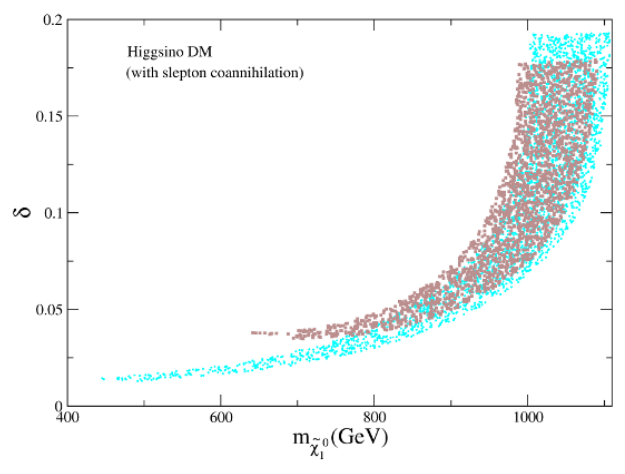

(a)

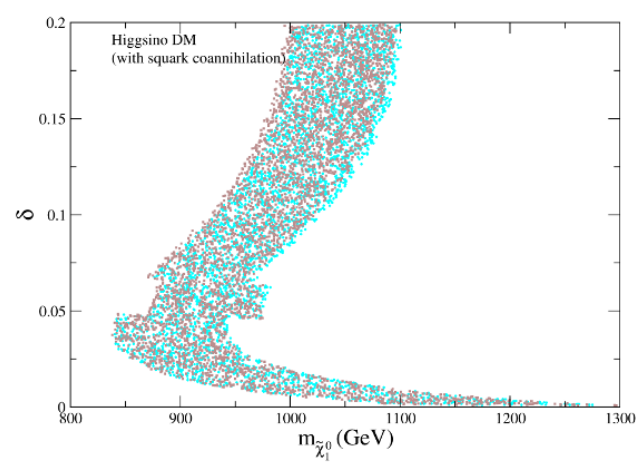

(b)

Figure 2. a) Scatter plot for the mass of LSP $m_{\widetilde{\chi}_{1}^{0}}$ vs $\delta$, the relative deviation of slepton masses with respect to the mass of the LSP for the LSP-slepton coannihilation scenarios including also sneutrinos, where the LSP is highly higgsino dominated in its composition. For a given slepton mass labeled as $m_{\tilde{l}_{i}}$ the relative deviation is given as $\delta_{i}=\frac{\left(m_{\tilde{l}_{i}}-m_{\tilde{\chi}_{1}^{0}}\right)}{m_{\tilde{\chi}_{1}^{0}}} . \delta$ refers to the average of the values of $\delta_{i}$ for all the three generations of sleptons including all the internal degrees of freedom of sleptons as mentioned in section 1. The two different colors namely cyan (circle) and brown (square) refer to the cases of $\tan \beta=10$ and 30 respectively. b) The results of considering the coannihilations of LSP with the first two generations of squarks for $\tan \beta=10$ and 30 . The color scheme is similar to (a).

the third generation of sleptons (section 2). A relatively larger spread of stau masses via exponential suppression effectively reduces the corresponding coannihilation contributions toward the effective cross section. The cases of no slepton coannihilations hardly depend on $\tan \beta$. This is consistent with the discussion made in section 2. We extend the results to LSP-squark coannihilations in figure 1(b). All the internal degrees of freedom are taken into account including the colors. The sleptons, on the other hand, are chosen to be very heavy $(3 \mathrm{TeV})$ as mentioned before. The color codes are same as those of figure 1(a). Here, squarks refer only to the first two generations for reasons mentioned in section 2 . For the lower limit of the LSP mass, one finds $m_{\widetilde{\chi}_{1}^{0}} \simeq 840 \mathrm{GeV}$ irrespective of $\tan \beta$. In any case, the above is rather a modest reduction from about $1 \mathrm{TeV}$ corresponding to the case of a generic higgsino DM. We must also note that for squark coannihilations the upper limit of $m_{\widetilde{\chi}_{1}^{0}}$ is stretched by about $200 \mathrm{GeV}$ corresponding to the no sfermion coannihilation case. In this zone of large LSP mass, the relic density decreases compared to the generic higgsino DM case due to the dominance of squark-squark coannihilations. We will come back to it for further discussion while describing figure 2(b).

Figure 2 shows scattered points in the $m_{\widetilde{\chi}_{1}^{0}}-\delta$ plane corresponding to the analysis of figure 1. Figure 2(a) shows the scatter plot in the $\left(m_{\widetilde{\chi}_{1}^{0}}-\delta\right)$ plane for LSP coannihilating with sleptons. The points that correspond to satisfying the DM relic density limits of eq. (3.1) have two different colors namely cyan (circle) and brown (square) representing the cases of $\tan \beta=10$ and 30 respectively. The left and right hand side white regions indicate LSP to be underabundant and overabundant respectively. The regions with large 
$\delta$ are associated with smaller degrees of coannihilation. The brown region corresponding to $\tan \beta=30$ has a larger degree of stau L-R mixing. This follows from the discussion made in section 2. Thus, compared to $\tan \beta=30$, the effect of slepton coannihilations is more prominent, thereby meaning the lower limit of the LSP mass to become smaller for $\tan \beta=10$. As seen in the figure, this leads to a higgsino dominated LSP with mass as low as $450 \mathrm{GeV}$ satisfying eq. (3.1). The associated coannihilating sleptons correspond to $\delta$ less than $2 \%$. We also note that as explained in section 2 as well as in the description of figure 1(a), an analysis with only the first two generations of sleptons would hardly show any dependence on $\tan \beta$ concerning the lower limit of the LSP mass satisfying the relic density constraint of eq. (3.1). Figure 2(b) shows the result for the LSP-squark coannihilations where we have considered only the first two generations of squarks for which the outcome is essentially independent of $\tan \beta$. On the lower side, the LSP mass satisfying eq. (3.1) is reduced to around $840 \mathrm{GeV}$ from $1 \mathrm{TeV}$ corresponding to the generic higgsino DM case. On the other end, the higgsino LSP mass may extend to about $1300 \mathrm{GeV}$, about a $20 \%$ increase than the generic higgsino DM upper limit. There is a "notch" region corresponding to $\delta \sim 0.05$ spreading across the values of the LSP mass. In this quasi degenerate LSPsquark setup, the above arises due to a relatively rapid change of the DM relic density coming out of the enhancement of $q \tilde{q} \tilde{\chi}_{1,2}^{0}$ effective couplings. We will discuss this at the end of section 4.1 .

In the zone of relatively large LSP mass and for nearly degenerate squarks and the LSP i.e. small values of $\delta$, a detail check of the outgoing products of annihilation and coannihilations confirms that the squark-squark coannihilations dominate over the generic higgsino DM effective annihilation cross-section. We note that the latter, which is inversely proportional to the DM relic density, decreases with increase in higgsino mass. ${ }^{4}$ Additionally, for larger values of $\delta$ and larger LSP mass, in spite of a smaller degree of generic electroweakino annihilation/coannihilations due to heavier LSP, the squark-squark coannihilations are more and more exponentially suppressed. Thus, even for slightly larger values of $\delta$ we get overabundance of DM.

The result of a combined analysis of the above slepton (three generations) and squark coannihilations (two generations) is given in figure 3. The colors refer to the same convention as that of figure 2 . The top-squark masses are chosen to be very heavy $(3 \mathrm{TeV})$. The left and right hand side blanck regions indicate LSP to be underabundant and overabundant respectively. Here, a large number of sfermions participate in coannihilations and the region with small LSP mass that would have otherwise underabundant DM gets the right amount of relic density even for relatively larger values of $\delta$. The relic density is clearly enhanced thus satisfying eq. (3.1). However, among a variety of participating coannihilation channels, predominant contributions still come from electroweakino coannihilations.

\footnotetext{
${ }^{4}$ For the generic higgsino LSP case one has $\Omega_{\widetilde{\chi}_{1}^{0}} h^{2}=0.10\left(\frac{\mu}{1 \mathrm{TeV}}\right)^{2}$ [73], where $\mu$ is given in TeV. A similar relation for a wino LSP with mass $m_{\tilde{W}}$ reads $\Omega_{\tilde{W}} h^{2}=0.13\left(\frac{m_{\tilde{W}}}{2.5 \mathrm{TeV}}\right)^{2}=0.021 m_{\tilde{W}}^{2}$ [73], denoting a factor of 5 stronger effective annihilation cross section compared to the higgsino case. As we will see the squark-squark coannihilation contributions are not large enough to supersede the generic wino DM depletion cross section. Hence, the wino dominated LSP scenario with squark coannihilations will not encounter any stretching of the LSP mass region satisfying the relic density data on the higher side.
} 


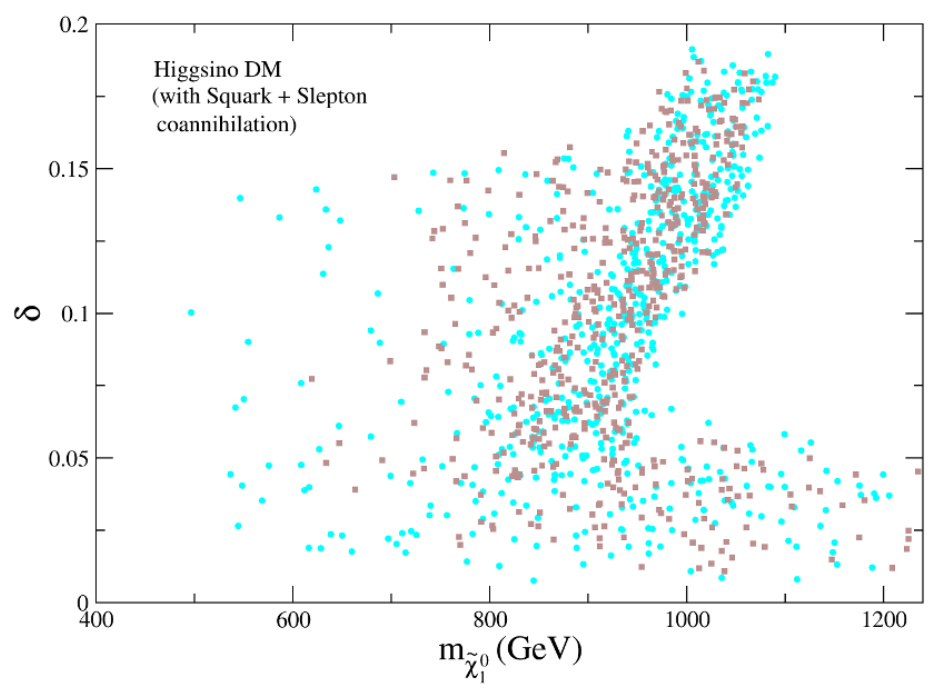

Figure 3. The results of a combined analysis of slepton and squark coannihilations at $20 \%$ level as mentioned in the text. The cyan (circle) and brown (square) points refer to $\tan \beta=10$ and 30 respectively. All the points in the scatter plot satisfy the lower and upper limits of DM relic density of eq. (3.1). The lower limit of the higgsino LSP mass satisfying the DM relic density constraint is around $500 \mathrm{GeV}$.

The lower limit of the higgsino LSP mass satisfying the DM relic density constraint is around $500 \mathrm{GeV}$.

\subsection{Wino dominated LSP}

A wino-like LSP in MSSM implies nearly degenerate $\widetilde{\chi}_{1}^{0}$ and $\widetilde{\chi}_{1}^{ \pm}$, both of whose masses being essentially determined by the $\mathrm{SU}(2)$ gaugino mass parameter $M_{2}$. The smallness of mass difference between $m_{\widetilde{\chi}_{1}^{0}}$ and $m_{\widetilde{\chi}_{1}^{ \pm}}$leads to intense coannihilations resulting into a relic density too low to satisfy the observed limits unless the mass of wino is too large (above $2 \mathrm{TeV}$ ). A choice like $M_{1}>\mu>M_{2}$ would give rise to a wino dominated LSP. In particular, we choose $\mu=2 M_{2}$ and $M_{1}>2.4 M_{2}$, so as to make the LSP predominantly a wino. $M_{2}$ is then varied in the range $100 \mathrm{GeV}<M_{2}<2.5 \mathrm{TeV}$. For slepton coannihilations and a given value of $M_{2}$, the common mass parameter for the slepton masses of all the three generations are varied within the range $50 \%$ below and above the value of $M_{2}$. The common squark mass parameters are pushed to $4 \mathrm{TeV}$. On the other hand, for squark coannihilations, the common squark mass parameter of the first two generations are similarly chosen around the value of $M_{2}$, while the latter being scanned as before. Here, the slepton mass parameters are large $(4 \mathrm{TeV})$. The squark masses of the third generation and the $\mathrm{SU}(3)$ gaugino mass parameter $M_{3}$ are kept at $4 \mathrm{TeV}$ while the CP-odd Higgs mass is set at $6 \mathrm{TeV}$ throughout our analysis, thus ensuring no s-channel Higgs resonance annihilations. Once again, owing to the variation of $M_{2}$ that results into varying $\mu$ we scan $A_{t}$ between $-2 \mathrm{TeV}$ to $-7 \mathrm{TeV}$ so as to have the higgs mass $m_{h}$ in the correct range. It can be observed from figure 4 that in the absence of any slepton coannihilation the relic 


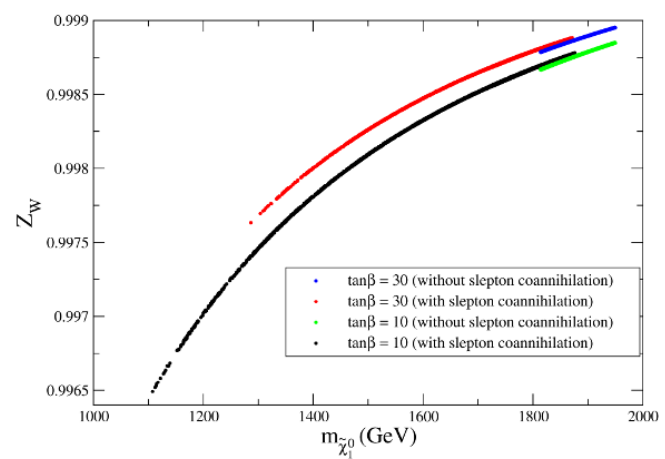

(a)

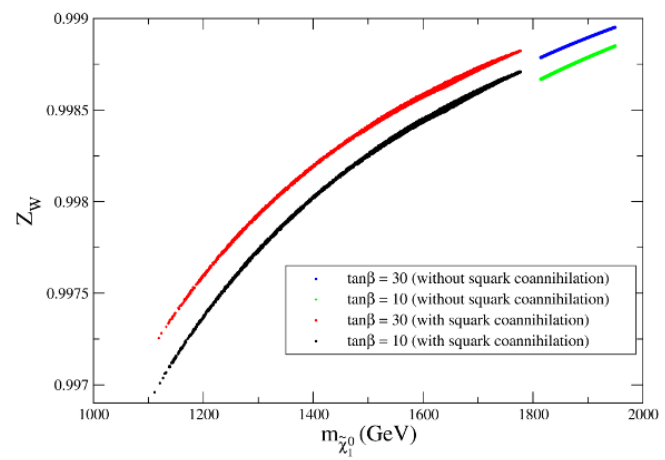

(b)

Figure 4. a) Plot of wino fraction $Z_{W}$ vs LSP mass for a wino dominated LSP when the LSP coannihilates with sleptons and sneutrinos of all the three generations apart from the usual LSP- $\widetilde{\chi}_{1}^{ \pm}$ coannihilations. The cyan (circle) and brown (square) points refer to $\tan \beta=10$ and 30 respectively. All the points that are generated by varying $M_{2}$ satisfy the lower and upper limits of DM relic density of eq. (3.1). Clearly, the slepton coannihilations reduce the lower limit of the LSP mass satisfying the WMAP/PLANCK data to $m_{\widetilde{\chi}_{1}^{0}} \simeq 1.1 \mathrm{TeV}$. b) Similar plot with LSP-squark coannihilations along with reference cases with no LSP-squark coannihilations where squarks refer only to the first two generations. The color codes are same as those of (a).

density becomes viable for $m_{\widetilde{\chi}_{1}^{0}} \sim 1.8 \mathrm{TeV} .{ }^{5}$ The presence of sleptons with masses close to $m_{\widetilde{\chi}_{1}^{0}}$ leads to many new coannihilation channels and affects the averaging procedure toward the effective cross section and as we will see this increases the DM relic density so that eq. (3.1) is satisfied for much smaller masses of the LSP.

The amount of the wino component in the LSP is expressed in terms of the wino fraction defined as, $Z_{W}=N_{12}^{2}$. The wino fractions for different LSP masses with/without sfermion coannihilations are shown in figure 4. We only show the parameter points that satisfy eq. (3.1) for the limits of DM relic density. Figure 4(a) shows the scatter plot of the wino fraction $Z_{W}$ vs LSP mass when the LSP efficiently coannihilates with sleptons and sneutrinos of all the three generations. We note that the LSP-sfermion coannihilations take place in the background of strong electroweakino coannihilations. The significance of wino purity level in turn is directly related to the level of coannihilations between the

\footnotetext{
${ }^{5}$ We must note that a wino mass of $1.8 \mathrm{TeV}$ satisfying the relic density data is low compared to what is seen in the literature, typically above $2 \mathrm{TeV}$. Obtaining a heavier wino that satisfies the DM relic density limits is possible i) via considering larger sfermion mass and $\mu$ as explained in the text in section 3.1 and ii) most importantly, via including non-perturbative effects like Sommerfeld correction. Sommerfeld correction is known to increase the wino mass that satisfies the relic density limits. We have not included such an effect particularly for the fact that a low wino mass like $1.1 \mathrm{TeV}$ would hardly have an appreciable degree of Sommerfeld effect. We would like to mention ref. [81] (their figure 2) and ref. [82] (their figure 2) in support of the smallness of the correction for our relevant zone of wino mass. Considering the fact that the relic density $\propto M_{2}^{2}$, using ref. [82] we estimate a 10-12\% level of enhancement of $M_{2}$ for its lower bound that would satisfy the observational relic density limits. Thus, the lower limit of the wino mass is estimated to change from $1.1 \mathrm{TeV}$ to around $1.2 \mathrm{TeV}$ (as we will come across in figure 4 ) if we include the Sommerfeld effect.
} 
electroweakino states $\widetilde{\chi}_{1}^{0}$ and $\widetilde{\chi}_{1}^{ \pm}$. As before, we have considered a maximum of $20 \%$ deviation in masses for the coannihilating particles with respect to the mass of the LSP. The squark masses of the first two generations are kept at $4 \mathrm{TeV}$. The reference results with no slepton coannihilations are shown in green and blue points for $\tan \beta=10$ and 30 respectively. The black and red points refer to $\tan \beta=10$ and 30 respectively for the cases with slepton coannihilations. Clearly, as seen in figure 4(a), the slepton coannihilations reduce the lower limit of the LSP mass satisfying eq. (3.1) to $m_{\widetilde{\chi}_{1}^{0}} \simeq 1.1 \mathrm{TeV}$ and $1.3 \mathrm{TeV}$ respectively for $\tan \beta=10$ and 30 . There is a dissimilarity in the lower limits in the results for the two different values of $\tan \beta$ for similar reasons as in the case of higggsino LSP mentioned in section 3.2. We extend the results to LSP-squark coannihilations in figure 4(b). Here the sleptons are chosen to be very heavy $(4 \mathrm{TeV})$. The color codes are same as those of figure 4(a). The squarks again refer only to the first two generations for reasons mentioned earlier. One finds the lower limit as $m_{\widetilde{\chi}_{1}^{0}} \simeq 1.1 \mathrm{TeV}$ for both values of $\tan \beta$.

Figure 5 shows the average degree of mass difference among the coannihilating partners while considering the coannihilations of LSP separately with sleptons or squarks where the LSP is wino dominated in its composition. This refers to the scanning corresponding to figure 4. The colored points belong to parameter space that satisfy eq. (3.1). Figure 5(a) shows the scatter plot in the $\left(m_{\widetilde{\chi}_{1}^{0}} \delta\right)$ plane. Apart from the sleptons we also include the three generations of sneutrinos in this analysis. $\delta$ is similarly defined as in the higgsino case of section 3.2. The color codes are same as those of figure 2. The left and right hand side white regions indicate LSP to be underabundant and overabundant respectively. The regions with large $\delta$ refer to smaller degrees of coannihilation because of larger exponential suppression. The brown region corresponding to $\tan \beta=30$ is associated with a larger degree of stau L-R mixing. Demanding both $\tilde{\tau}_{1}$ and $\tilde{\tau}_{2}$ along with the first two generations of sleptons to have masses within $20 \%$ of the LSP mass restricts the reach of $\delta$ for reasons mentioned in section 2. The effect of slepton coannihilations is more prominent for $\tan \beta=$ 10 and this leads to a wino dominated LSP having the right abundance with mass as low as $1.1 \mathrm{TeV}$ when the coannihilating sleptons have $\delta$ less than $2 \%$. Similar to the higgsino analysis, the $\tan \beta$ dependence of the lower limit of $m_{\widetilde{\chi}_{1}^{0}}$ satisfying eq. (3.1) would cease to exist if we had excluded the third generation of sleptons to take part in coannihilations. Figure 5(b) shows a similar result for the LSP-squark coannihilations where we consider only the first two generations of squarks as before. Clearly, being devoid of any top-squark coannihilations the result is essentially independent of $\tan \beta$. The lowest LSP mass that satisfies the DM relic density constraint is around $1.1 \mathrm{TeV}$. Unlike the higgsino case, there is no dominance of squark-squark coannihilations over the parameter space that satisfies the DM relic density constraint. This is indeed related to the large annihilation cross section that a wino has compared to that of a higgsino for a given mass of the LSP (see footnote\# 4). Similar to the higgsino case, there is a "notch" region corresponding to $\delta \sim 0.05$ spreading across the values of the LSP mass. In this quasi degenerate LSP-squark setup, the above arises due to a relatively rapid change of the DM relic density coming out of the enhancement of $q \tilde{q} \tilde{\chi}_{1}^{0}$ effective coupling. We will discuss this at the end of section 4.1.

The results of a combined analysis of the above slepton (three generations) and squark coannihilations (two generations) is given in figure 6 . The two different colors namely cyan 


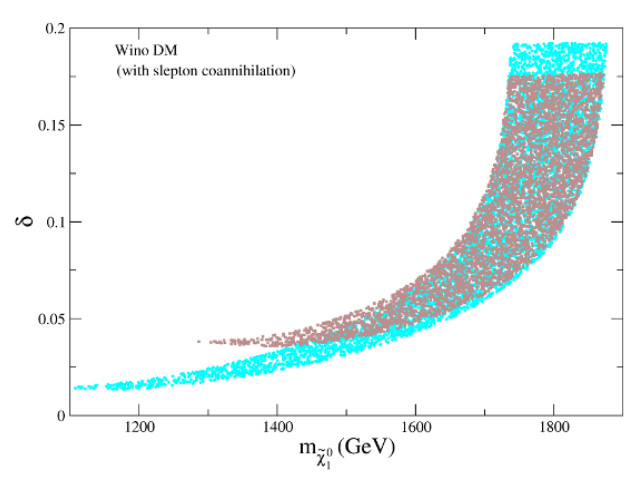

(a)

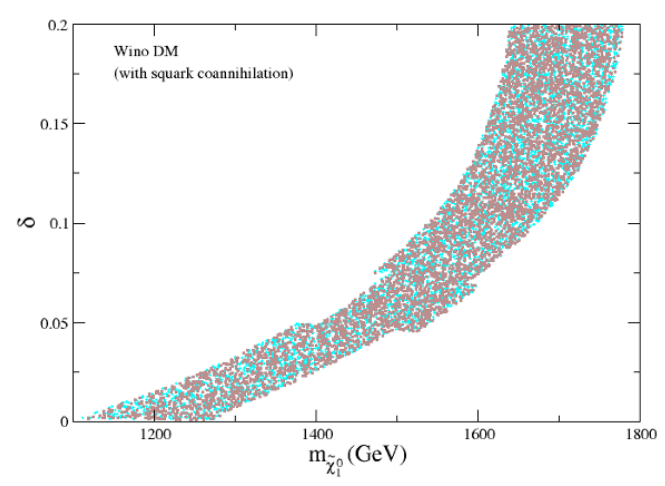

(b)

Figure 5. a) Scatter plot for the mass of LSP $m_{\widetilde{\chi}_{1}^{0}}$ vs $\delta$, the relative deviation of slepton masses with respect to the mass of the LSP for LSP-slepton coannihilation scenarios including also sneutrinos, where the LSP is highly wino dominated in its composition. For a given slepton mass labeled as $m_{\tilde{l}_{i}}$ the relative deviation is given as $\delta_{i}=\frac{\left(m_{\tilde{l}_{i}}-m_{\tilde{\chi}_{1}^{0}}\right)}{m_{\tilde{\chi}_{1}^{0}}} . \delta$ refers to the average of the values of $\delta_{i}$ for all the three generations of sleptons including all the internal degrees of freedom of sleptons as mentioned in section 1 . The two different colors namely cyan (circle) and brown (square) refer to the cases of $\tan \beta=10$ and 30 respectively. b) The results of considering the coannihilations of LSP with the first two generations of squarks for $\tan \beta=10$ and 30 . The color scheme is similar to (a).

(circle) and brown (square) refer to the cases of $\tan \beta=10$ and 30 respectively. The left and right hand side white regions indicate LSP to be underabundant and overabundant respectively. The regions with large $\delta$ refer to smaller degrees of coannihilation. The lower limit of the wino LSP mass satisfying the DM relic density constraint is around $900 \mathrm{GeV}$.

We would like to mention here that the ATLAS and CMS collaborations presented their results for chargino searches in the high transverse momentum $\left(p_{T}\right)$ disappearing tracks $[178,179]$ and long lived particle search channels for nearly degenerate $\widetilde{\chi}_{1}^{ \pm}$and $\widetilde{\chi}_{1}^{0}[180,181]$. The mass range of $\widetilde{\chi}_{1}^{ \pm}$considered in this analysis is well within these bounds.

\section{Direct and indirect detection of DM}

In this section we will probe the prospect of direct and indirect detection of the lightest neutralino. We will particularly come across the importance of squark exchange diagrams in computing the SI direct detection cross section. The squark exchange diagrams are usually less important since the Higgs exchange diagrams typically dominate. As we will see, in this analysis we are in a different situation because of considering quasi-degenerate squarks and LSP for the requirement of coannihilations.

\subsection{Direct detection}

Direct detection of DM involves finding the recoil energy deposited when a DM particle scatters off a detector nucleus [9, 10]. Spin-independent LSP-proton scattering may take place through s-channel squark exchange and t-channel Higgs exchange diagrams. 


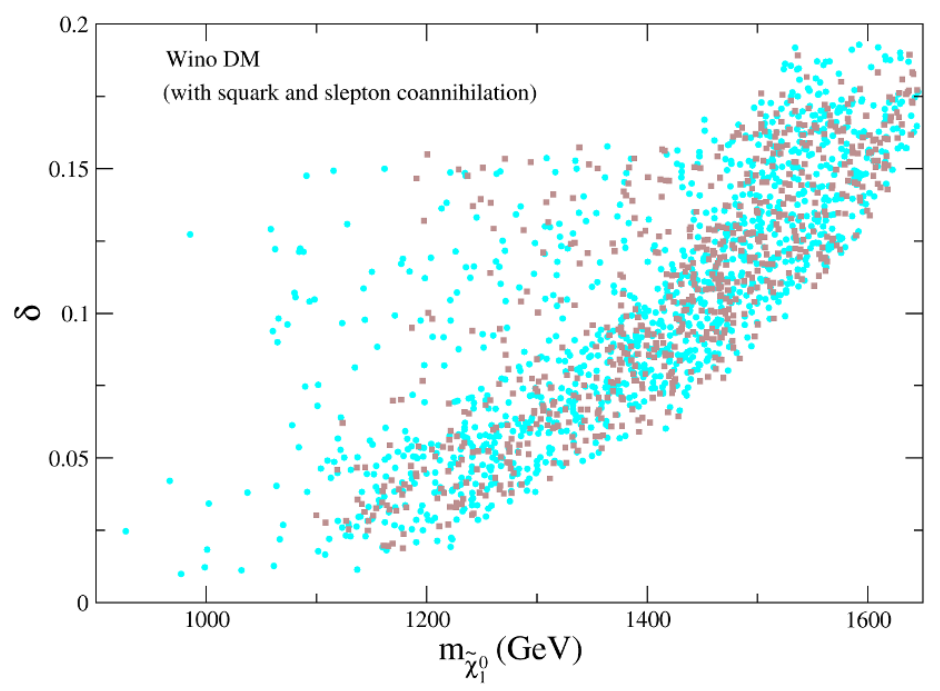

Figure 6. The results of a combined analysis of slepton and squark coannihilations at $20 \%$ level as mentioned in the text. The cyan (circle) and brown (square) points refer to $\tan \beta=10$ and 30 respectively. All the points in the scatter plot satisfy the lower and upper limits of DM relic density of eq. (3.1). The lower limit of the wino LSP mass satisfying the DM relic density constraint is around $900 \mathrm{GeV}$.

When the squarks are considerably heavy, the Higgs exchange diagrams typically dominate $[182,183] .^{6}$

The Higgs- $\widetilde{\chi}_{1}^{0}-\widetilde{\chi}_{1}^{0}$ coupling for the higgsino-LSP case can be written down in the form [146]:

$$
\begin{aligned}
C_{h \tilde{\chi} \tilde{\chi}} & \simeq \mp \frac{1}{2} M_{Z} c_{W}[1 \pm \sin 2 \beta]\left[\frac{t_{W}^{2}}{M_{1}-|\mu|}+\frac{1}{M_{2}-|\mu|}\right], \\
C_{H \tilde{\chi} \tilde{\chi}} & \simeq \frac{1}{2} M_{Z} c_{W} \cos 2 \beta\left[\frac{t_{W}^{2}}{M_{1}-|\mu|}+\frac{1}{M_{2}-|\mu|}\right],
\end{aligned}
$$

where $t_{W}=\tan \theta_{W}$ etc. with $\theta_{W}$ being the Weinberg angle. Similarly, for the wino-LSP case, the couplings are as follows [146]:

$$
\begin{aligned}
C_{h \tilde{\chi} \tilde{\chi}} & \simeq \frac{M_{Z} c_{W}}{M_{2}^{2}-\mu^{2}}\left[M_{2}+\mu \sin 2 \beta\right], \\
C_{H \tilde{\chi} \tilde{\chi}} & \simeq-\frac{M_{Z} c_{W}}{M_{2}^{2}-\mu^{2}} \mu \cos 2 \beta .
\end{aligned}
$$

From the above expressions it is clear that the couplings and hence the scattering cross-section would be large if there is a large degree of mixing between the gaugino and the higgsino components of the LSP. We also note that couplings become weaker for increased gaugino masses and $\mu$. On the other hand, a pure higgsino or a wino LSP with

\footnotetext{
${ }^{6}$ On the contrary, we will soon discuss the scenario when the squark exchange diagrams may even dominate over the Higgs exchange diagrams.
} 


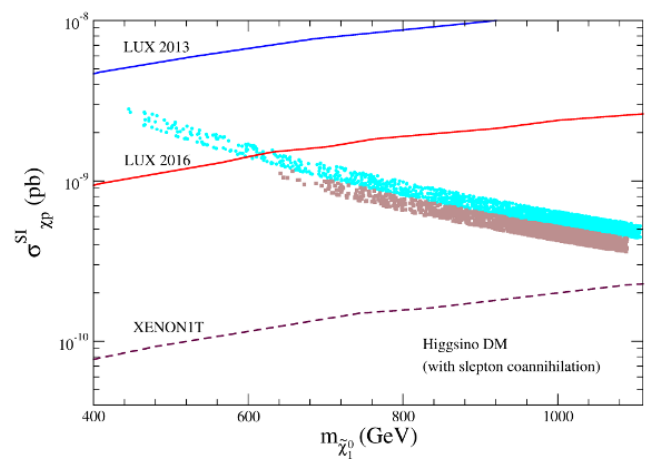

(a)

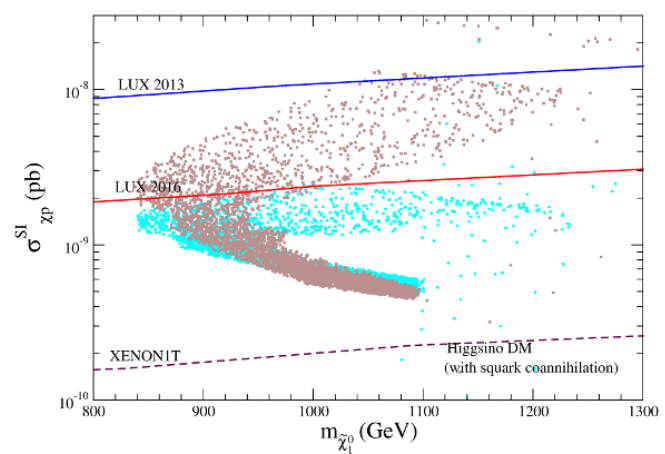

(b)

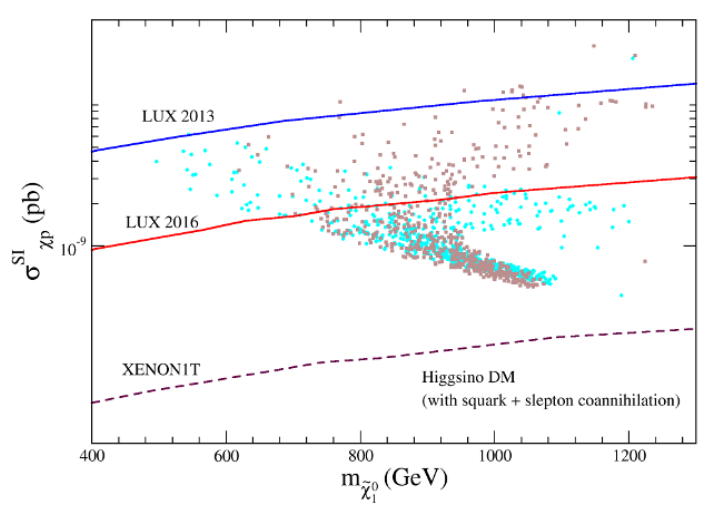

(c)

Figure 7. (a) Scatter plot of spin-independent DM direct detection cross-section vs LSP mass for higgsino dominated LSPs undergoing LSP-slepton coannihilations. The cyan and brown points represent $\tan \beta=10$ and 30 respectively that satisfy eq. (3.1). The red line (solid) is the LUX 2016 exclusion contour and the maroon dashed line shows the expected limit from the future XENON1T experiment. (b) Same as (a) except LSP undergoing LSP-squark coannihilations. (c) Same as (a) except LSP undergoing slepton plus squark coannihilations.

very little mixing can hardly be able to produce large values of spin-independent crosssection. Figure 7 shows our results for DM direct SI detection cross-section where only the points satisfying eq. (3.1) are shown for a higgsino type of LSP undergoing LSP-slepton coannihilations (figure 7(a)) and LSP-squark coannihilations (figure 7(b)). The cyan and brown points correspond to $\tan \beta=10$ and 30 respectively. The red line (solid) is the LUX 2016 exclusion contour [145] and the maroon dashed line shows the expected limit from the future XENON1T experiment [184]. Clearly, the recent LUX data rules out low higgsino mass region below $600 \mathrm{GeV}$ (figure $7(\mathrm{a})$ ). We must also remember the existence of uncertainty, around one order in magnitude, in the computation of the SI direct detection cross-section. Factors like strangeness content of nucleon, local DM density, velocity distribution profiles, all contribute toward such uncertainty amount (see ref. [113] and references therein). As seen in figure 7(a) the higgsino LSP scenario may be effectively probed via 
XENON1T. Figure 7(b) shows similar results for the LSP-squark coannihilations. Here, the lowest LSP mass that survives after the LUX 2016 data is about $840 \mathrm{GeV}$. Additionally, a large region of parameter space $^{7}$ is discarded via the same experiment without however affecting the lowest possible value of the LSP mass. Compared to the case of figure 7(a) here the SI direct detection cross-sections are generally large. This is a signature of having a quasi-degenerate squark and LSP scenario that comes into our study of the LSP-squark coannihilations. Here the effective coupling constant for quark-LSP scattering drastically increases [185] causing the cross-section to be larger, often much more than the LUX limit for a significant zone of the LSP mass. Thus, the squark exchange diagrams are potentially able to compete with or even dominate over the Higgs exchange diagrams while contributing to the total direct detection cross-section. ${ }^{8}$ This is of course true for some region of parameter space where the degree of the LSP-squark mass degeneracy is higher. We remind that the above is unlike the usually encountered MSSM parameter regions where Higgs exchange diagrams dominate over the squark exchange diagrams in the SI direct detection cross-section. Coming back to figure 7(b) we see that a lot of parameter space is eliminated via LUX 2016 data. The remaining parameter space can fully be probed in the XENON1T experiment. The effect of including both slepton and squark coannihilations is shown in figure $7(\mathrm{c})$. The lowest LSP mass limit satisfying the LUX data is around $680 \mathrm{GeV}$.

Figure 8 shows our results for DM direct SI detection cross-section where only the points satisfying eq. (3.1) are shown for a wino type of LSP undergoing slepton coannihilations (figure 8(a)) and squark coannihilations (figure 8(b)). The cyan and brown points correspond to $\tan \beta=10$ and 30 respectively. The red line (solid) is the LUX 2016 exclusion contour [145] and the maroon dashed line shows the expected limit from the future XENON1T experiment [184]. Clearly, as seen in figure 8(a) the cross-section is too low so that even XENON1T would not be able to probe this scenario except around the $1.1 \mathrm{TeV}$ region for LSP mass. We must additionally clarify that a comparison of figure 7 (a) and figure 8(a) shows that contrary to what we would naively expect, the SI cross-section in the latter case is in general smaller. The reason lies in the fact that the values of $m_{\widetilde{\chi}_{1}^{0}}$ that satisfy the relic density limits for a wino like LSP are much higher than that of a higgsino dominated LSP. Figure 8(b) shows similar results for the LSP-squark coannihilations. Here, the lowest LSP mass that survives after the LUX 2016 data is about $1.27 \mathrm{TeV}$. Additionally, a large region of parameter space is discarded via the same experiment while eliminating a window of $1.1 \mathrm{TeV}$ to $1.27 \mathrm{TeV}$ of LSP mass. XENON1T would not be able to probe this scenario except the region close to $1.2 \mathrm{TeV}$ of the LSP mass. Similar to the case of higgsino-squark coannihilations, the SI direct detection cross-section is much larger for wino-squark coannihilation scenario compared to the wino-slepton results. The squark exchange contributions can be significantly large for the parameter points associated with near degeneracy of the squark and LSP masses as explained before. Here, we observe that the cross-section can be quite large even for squark masses $\gtrsim 1.2 \mathrm{TeV}$. The Higgs-exchange contributions are sub-dominant in this case of wino-dominated LSP that has a very small

\footnotetext{
${ }^{7}$ By parameter space one really means here a smeared region of squark masses around a given LSP mass.

${ }^{8}$ The extent of any cancellation effect on the contrary is small, unlike the wino scenario that we will see soon.
} 


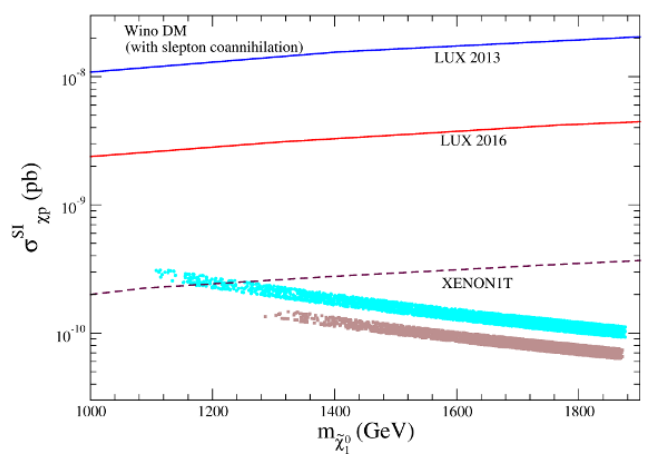

(a)

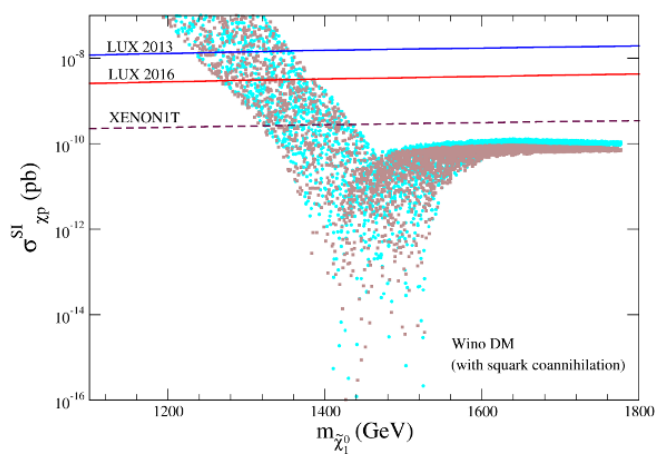

(b)

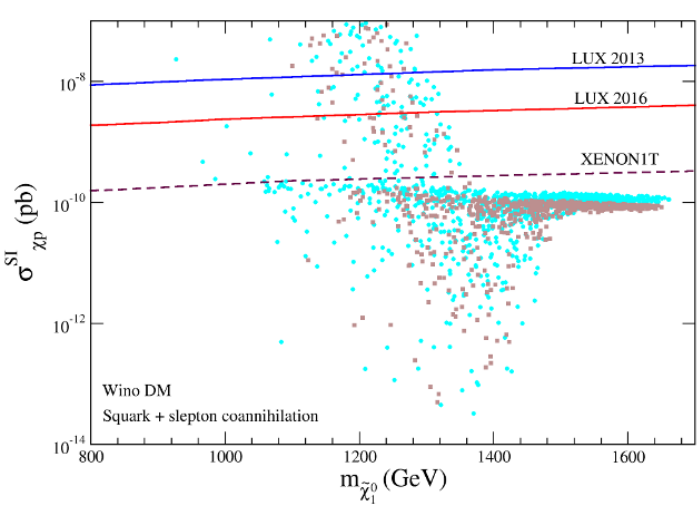

(c)

Figure 8. (a) Scatter plot of spin-independent DM direct detection cross-section vs LSP mass for wino dominated LSPs undergoing LSP-slepton coannihilations. The cyan and brown points represent $\tan \beta=10$ and 30 respectively that satisfy eq. (3.1). The red line (solid) is the LUX 2016 exclusion contour and the maroon dashed line shows the expected limit from the future XENON1T experiment. (b) Same as (a) except LSP undergoing LSP-squark coannihilations. (c) Same as (a) except LSP undergoing slepton plus squark coannihilations.

higgsino content. We must also note that for a fixed value of $m_{\widetilde{\chi}_{1}^{0}}$ we get a wide degree of variation in cross-section with some points exceeding the LUX limit whereas some points having values below the limit. In the latter case there is a cancellation among the contributions from the squark and Higgs exchange diagrams that pushes the cross-section to very low values. Similar to what happens for the higgsino LSP case as in figure 7(b), this is a signature of quasi-degenerate squarks and LSP that leads to a large increase in the effective coupling constant for quark-LSP scattering [185].

Finally, the effect of including both slepton and squark coannihilations is shown in figure 8(c). The lowest LSP mass limit satisfying the LUX data is about $1 \mathrm{TeV}$.

We will come to the discussion of the notch regions of figure 2(b) and figure 5(b). Apart from direct detection, enhancement of $q \tilde{q} \tilde{\chi}$ effective coupling near the degenerate zone of squarks and LSP masses has its important signature also on the DM relic density. For a 
wino dominated LSP that does not have a quasi degenerate neutralino state, the notch region is found to coincide with the mentioned cancellation region of $\sigma_{\chi p}^{S I}$ (i.e. cancellation between the higgs exchange and the squark exchange diagrams). Corresponding to a given mass of the LSP, this is the region of $\delta$ where the above effective coupling becomes large. The situation for a higgsino-LSP case is more involved. This is principally because on the top of the coannihilations a wino LSP would undergo, there are additional coannihilation processes like $\widetilde{\chi}_{2}^{0} \widetilde{\chi}_{1}^{0}, \widetilde{\chi}_{2}^{0} \tilde{q}$ contributing toward the higgsino relic density. As a result, in spite of a cancellation zone of $\sigma_{\chi p}^{S I}$ for certain values of $\delta$, coannhilation effects potentially smear the abrupt change in the higgsino relic density coming out of the effect of enhanced $\widetilde{\chi}_{1}^{0} \tilde{q} q$ and $\widetilde{\chi}_{2}^{0} \tilde{q} q$ coupling strengths. Consequently, for a higgsino DM the values of $\delta$ that correspond to a cancellation or an enhancement zone in $\sigma_{\chi p}^{S I}$ are not the same where the anomalous "notch" zone of the relic density occurs. However, the enhancement of coupling remains a valid fact. It is seen that for a given $m_{\widetilde{\chi}_{1}^{0}}$ there is an abrupt decrease of the DM relic density corresponding to some range of $\delta$. Once a lower and a upper limit of the relic density are imposed, the above decrease in relic density irrespective of the LSP mass, leads to the formation of the notch regions for some effective range of values of $\delta$. Details may be explained by examining the relevant coupling enhancements as given in ref. [185]. ${ }^{9}$

\subsection{Indirect detection}

DM particles may get trapped due to gravity inside astrophysically dense objects like the Sun or the Earth by losing energy through repeated scattering with the nucleons. Inside the core of these objects DM particles may undergo pair annihilations leading to SM particles like fermion-antifermion pairs, gauge bosons etc. in the final state. The resulting antiparticles, neutrinos and gamma rays can offer interesting indirect signals of DM in the galaxy.

The high energy neutrinos produced as end products of DM pair annihilation in the solar core can produce muons through charged current interactions. The IceCube experiment [147] provides bounds on the muon flux for the pair annihilation channel DM DM $\rightarrow W^{+} W^{-}$. Figure 9(a) shows a scatter plot of the values of muon flux as a function of $m_{\widetilde{\chi}_{1}^{0}}$ for higgsino dominated LSPs undergoing slepton coannihilations for parameter points satisfying eq. (3.1). The cyan and brown points correspond to $\tan \beta=10$ and 30 respectively. The green and blue lines are the current and projected limits from the IceCube experiment $[147,148]$ in the $\widetilde{\chi}_{1}^{0} \widetilde{\chi}_{1}^{0} \rightarrow W^{+} W^{-}$channel. Similarly, figure 9 (b) shows the results for LSP-squark coannihilations. Clearly, the fluxes in both the above figures are in general too small to be probed.

The results of muon flux for the case of a wino dominated LSP is shown in figure 10. Figure 10(a) shows the results for LSP-slepton coannihilations for parameter points that satisfy eq. (3.1) for wino dominated LSPs undergoing slepton coannihilations. The color convention and the details of the limits from IceCube data are similar to figure 9(a). Clearly, the flux is too small to be probed for the LSP-slepton coannihilation scenario. Similarly, figure 10(b) shows the results for LSP-squark coannihilations. The result does not show

\footnotetext{
${ }^{9}$ Specifically we refer eqs. 15 to 17 and $\mathrm{A} 8$ to A14 of the paper.
} 


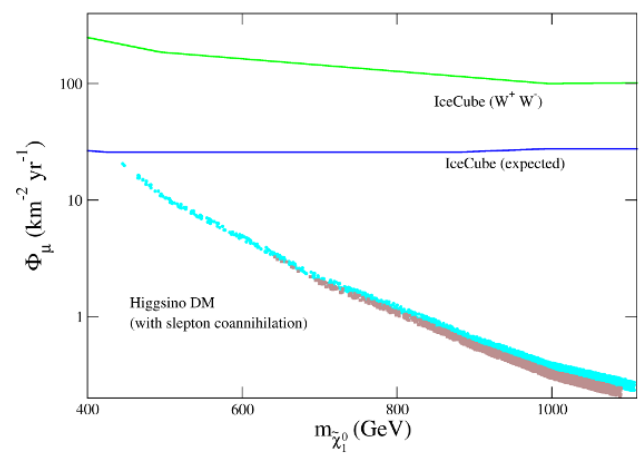

(a)

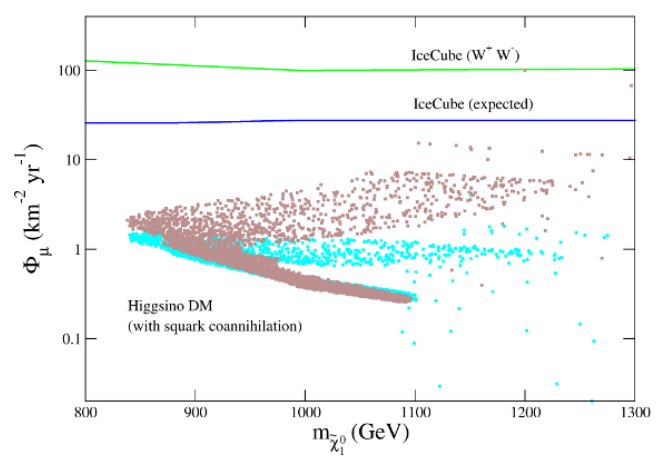

(b)

Figure 9. (a) Scatter plot of muon flux vs $m_{\widetilde{\chi}_{1}^{0}}$ for higgsino LSPs undergoing LSP-slepton coannihilations for $\tan \beta=10$ (cyan) and 30 (brown) while satisfying eq. (3.1). Present and future IceCube limits are shown as green and blue lines respectively. (b) same as (a) except the LSP is undergoing LSP-squark coannihilations.

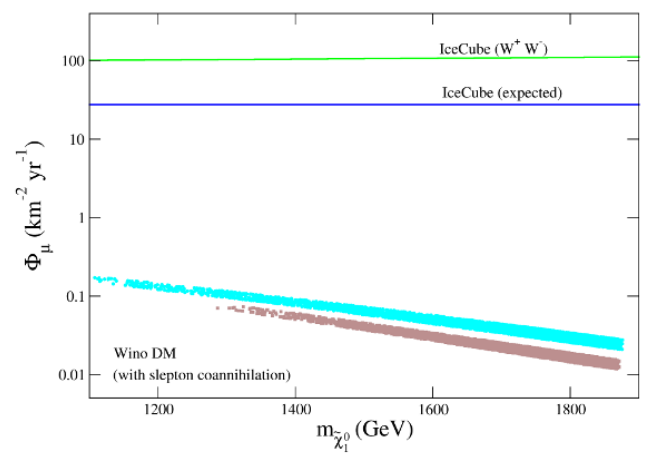

(a)

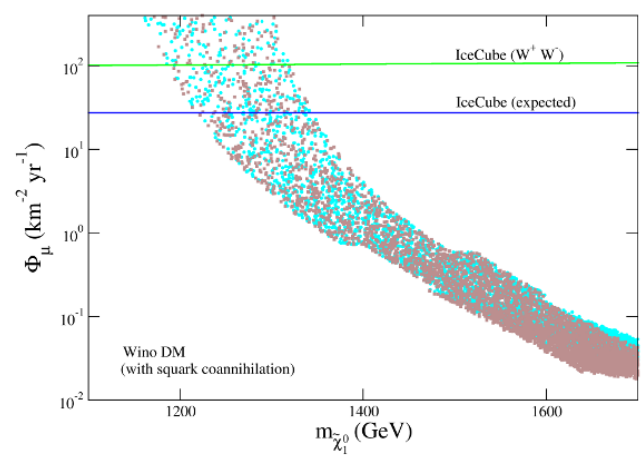

(b)

Figure 10. (a) Scatter plot of muon flux vs $m_{\widetilde{\chi}_{1}^{0}}$ for wino LSPs undergoing LSP-slepton coannihilations for $\tan \beta=10$ (cyan) and 30 (brown) while satisfying eq. (3.1). Present and future IceCube limits are shown as green and blue lines respectively. (b) same as (a) except the LSP is undergoing LSP-squark coannihilations.

any more exclusion of low mass LSP region compared to what is seen in figure 8(b) for the SI direct detection cross section.

Let us now discuss the constraints on spin-dependent DM-nucleon interaction crosssection as derived from the IceCube data. Inside the solar core, the number density $N$ of DM particles at any instant of time $t$ is obtained from the following [9],

$$
\frac{d N}{d t}=C_{c}-C_{A} N^{2}
$$

where, $C_{c}$ is the capture rate of DM by interaction with the nucleons present at the surface of the Sun whereas $C_{A}$ is related to the annihilation rate $\Gamma_{A}$ as: $\Gamma_{A}=\frac{1}{2} C_{A} N^{2}$. Solution of eq. (4.3) leads to $\Gamma_{A}=\frac{1}{2} C_{c} \tan h^{2}(t / \tau)$, with $\tau=\frac{1}{\sqrt{C_{c} C_{A}}}$. Hence, the capture rate is 


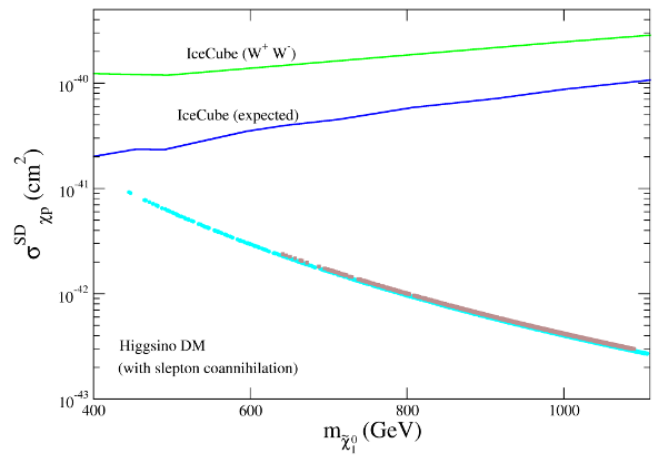

(a)

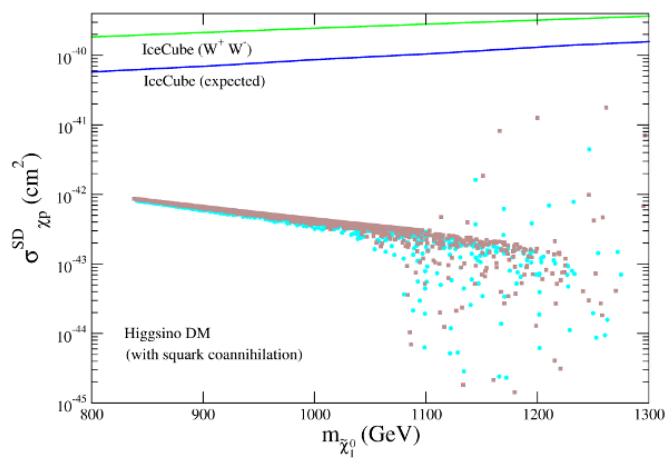

(b)

Figure 11. (a) Scatter plot of spin-dependent direct detection cross-section vs $m_{\widetilde{\chi}_{1}^{0}}$ for higgsino LSPs undergoing LSP-slepton coannihilations for $\tan \beta=10$ (cyan) and 30 (brown) while satisfying eq. (3.1). Present and future IceCube limits are shown as green and blue lines respectively. (b) Same as (a) except the LSP is undergoing LSP-squark coannihilations.

determined by the annihilation rate and when the age of the universe is much greater than $\tau$ (which occurs for large $C_{c}$ and $C_{A}$ ), an equilibrium is reached so that $\Gamma_{A}=\frac{1}{2} C_{c}$. Thus, it is possible to put bounds on the annihilation and capture cross-sections by looking at the indirect DM signals from the Sun. Since, capture of the DM particles occurs through spin-independent/dependent (SI/SD) DM interactions with the nucleons, these bounds get translated into the bounds on DM SI/SD interaction cross-sections.

$\widetilde{\chi}_{1}^{0}$ can have spin-dependent interaction with the quarks via s-channel squark exchange and t-channel $Z$-boson exchange processes. Similar to the SI case, while considering LSPslepton coannihilations, we can safely ignore the contributions from the squark exchange processes since the squarks are taken to be heavy. The tree level $Z \widetilde{\chi}_{1}^{0} \widetilde{\chi}_{1}^{0}$ coupling is given by $c_{Z \widetilde{\chi}_{1}^{0} \widetilde{\chi}_{1}^{0}}=\left(N_{13}^{2}-N_{14}^{2}\right)$. For the higgsino LSP case the coupling is given as [146],

$$
c_{Z \widetilde{\chi}_{1}^{0} \widetilde{\chi}_{1}^{0}} \simeq \mp \frac{1}{2}\left(t_{W}^{2} \frac{m_{W}^{2}}{M_{1} \mu}+\frac{m_{W}^{2}}{M_{2} \mu}\right) \cos 2 \beta+O\left(\frac{\mu}{M_{1}}, \frac{\mu}{M_{2}}\right),
$$

with $\mu>0(\mu<0)$. The same coupling for the wino case takes the form [146],

$$
c_{Z \widetilde{\chi}_{1}^{0} \widetilde{\chi}_{1}^{0}} \simeq \frac{m_{W}^{2}}{M_{2}^{2}-\mu^{2}} \cos 2 \beta .
$$

Thus, in general the couplings get suppressed as the LSP, irrespective of a higgsino or a wino becomes heavy. Figure 11(a) shows the results for the SD cross section for the higgsino dominated LSP scenario for LSP-slepton coannihilation. Figure 11(b) shows the LSP-squark coannihilation case for which the degeneracy between squark and the LSP masses (similar to what was described in the SI case) may push up the SD cross section. In general the IceCube limits would be inadequate to probe such higgsino models.

Figure 12 shows the results for the SD cross section for the wino dominated LSP scenario for LSP-slepton coannihilation (figure 12(a)) and LSP-squark coannihilation (figure 12(b)) cases. Although the IceCube limits may eliminate some region of parameter 


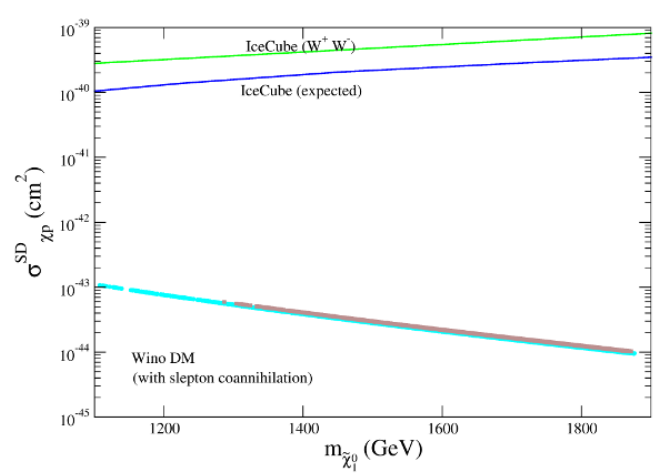

(a)

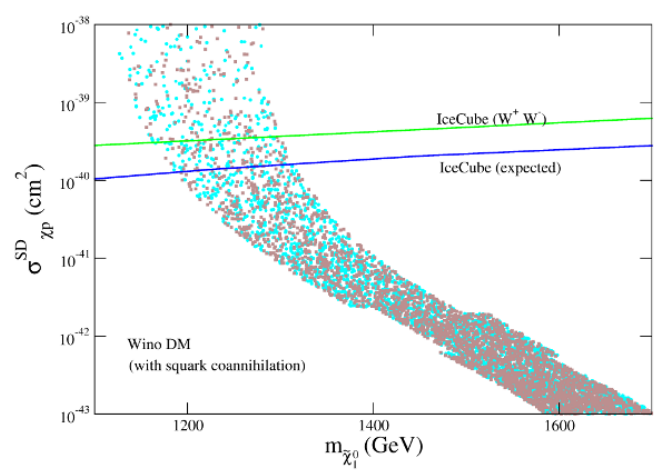

(b)

Figure 12. (a) Scatter plot of spin-dependent direct detection cross-section vs $m_{\widetilde{\chi}_{1}^{0}}$ for wino LSPs undergoing LSP-slepton coannihilations for $\tan \beta=10$ (cyan) and 30 (brown) while satisfying eq. (3.1). Present and future IceCube limits are shown as green and blue lines respectively. (b) Same as (a) except the LSP is undergoing LSP-squark coannihilations.

space where the LSP undergoes squark coannihilations, the result does not show any more exclusion of low mass LSP region compared to what is seen in figure 8(b) for the SI direct detection cross section.

In table 1 we show two benchmark points (BP) satisfying WMAP/PLANCK relic density limits of eq. (3.1) as well as the direct and indirect detection limits from the LUX and IceCube experiments respectively. BP1 and BP2 correspond to the case of a higgsino-LSP undergoing slepton and squark coannihilations for masses $617 \mathrm{GeV}$ and $760 \mathrm{GeV}$ respectively for $\tan \beta=10$. Monojet searches at the $14 \mathrm{TeV}$ LHC can probe pure higgsino scenario only upto the mass of $m_{\widetilde{\chi}_{1}^{0}} \sim 410 \mathrm{GeV}$ [186]. However, the situation looks more promising for a $100 \mathrm{TeV}$ collider where higgsinos may be probed upto $1.2 \mathrm{TeV}$. Existing disappearing track searches at the LHC do not have much sensitivity to a higgsino LSP. However, with modifications in search strategy, as suggested in refs. [187, 188], higgsinos upto $\sim 600 \mathrm{GeV}$ and $\sim 1.1 \mathrm{TeV}$ could be probed by the $14 \mathrm{TeV}$ high luminosity (HL)-LHC and a $100 \mathrm{TeV}$ collider respectively. BP3 and BP4 refer to wino-like LSP participating in slepton and squark coannihilations with masses $1011 \mathrm{GeV}$ and $1188 \mathrm{GeV}$ respectively for the same value of $\tan \beta$. Although a HL-LHC at $14 \mathrm{TeV}$ seems to be unable to probe these benchmark points, a $100 \mathrm{TeV}$ collider, with an exclusion reach of $\sim 1.8 \mathrm{TeV}$ in the monojet search channel can decisively explore such scenarios [186]. These benchmark scenarios for wino-like DM is likely to evade the HL-LHC even with disappearing track searches. However, the same searches at a $100 \mathrm{TeV}$ collider can conclusively probe these cases. Apart from collider searches, all the four BPs will be probed in near future with the XENON1T experiment. However, they are unlikely to produce any signal in future indirect detection experiments. 


\begin{tabular}{|c|c|c|c|c|}
\hline \multirow{2}{*}{$\begin{array}{c}\text { Parameters } \\
\text { Points }\end{array}$} & \multicolumn{2}{|c|}{ Higgsino DM } & \multicolumn{2}{|c|}{ Wino DM } \\
\hline & $\mathrm{BP} 1$ & $\mathrm{BP} 2$ & BP3 & $\mathrm{BP} 4$ \\
\hline$M_{1}(\mathrm{GeV})$ & 1221.5 & 1502.8 & 2339.2 & 2847.8 \\
\hline$M_{2}(\mathrm{GeV})$ & 1465.8 & 1803.4 & 974.7 & 1186.6 \\
\hline$\mu(\mathrm{GeV})$ & 610.8 & 751.4 & 1949.3 & 2373.2 \\
\hline$M_{\tilde{q}_{L, R}}(\mathrm{GeV})$ & 3000 & 539.6 & 4000 & 944.4 \\
\hline$M_{\tilde{l}_{L, R}}(\mathrm{GeV})$ & 628.4 & 3000 & 1026.3 & 4000 \\
\hline$m_{\widetilde{\chi}_{1}^{0}}(\mathrm{GeV})$ & 617.3 & 760.5 & 1011.1 & 1188.5 \\
\hline$m_{\widetilde{\chi}_{1}^{ \pm}}(\mathrm{GeV})$ & 620.4 & 763.1 & 1011.1 & 1188.5 \\
\hline$m_{\widetilde{\chi}_{2}^{0}}(\mathrm{GeV})$ & 1499 & 767 & 1969 & 2392 \\
\hline$m_{\widetilde{\chi}_{3}^{0}}(\mathrm{GeV})$ & 1198 & 1471.8 & 1969.8 & 2393 \\
\hline$M_{\tilde{(} e, \mu)_{L}}(\mathrm{GeV})$ & 630 & 3000 & 1027.2 & 4000 \\
\hline$M_{\tilde{(}_{e, \mu)_{R}}}(\mathrm{GeV})$ & 630 & 3000 & 1027.2 & 4000 \\
\hline$m_{\widetilde{\tau}_{1}}(\mathrm{GeV})$ & 621.7 & 2998 & 1011.1 & 3995.2 \\
\hline$m_{\widetilde{\tau}_{2}}(\mathrm{GeV})$ & 638 & 3000 & 1043 & 4005.2 \\
\hline$M_{\tilde{\nu}}(\mathrm{GeV})$ & 625.3 & 2999 & 1024.4 & 3999.5 \\
\hline$M_{\tilde{u}_{L}}(\mathrm{GeV})$ & 3000 & 792.8 & 4130.7 & 1228 \\
\hline$M_{\tilde{d}_{L}}(\mathrm{GeV})$ & 3000 & 796.8 & 4131.4 & 1230.5 \\
\hline$M_{\tilde{u}_{R}}(\mathrm{GeV})$ & 3000 & 793.7 & 4130.9 & 1228.5 \\
\hline$M_{\tilde{d}_{R}}(\mathrm{GeV})$ & 3000 & 795 & 4131.1 & 1229.4 \\
\hline$M_{\tilde{t}_{1}}$ & 2905.5 & 2889.0 & 3956.1 & 3962.6 \\
\hline$m_{h}$ & 126.5 & 126.4 & 126.5 & 125.9 \\
\hline$\Omega_{\tilde{\chi}} h^{2}$ & 0.126 & 0.092 & 0.091 & 0.09 \\
\hline$\sigma_{S I} \times 10^{-9}(\mathrm{pb})$ & 1.39 & 1.69 & 0.33 & 1.21 \\
\hline$\sigma_{S D} \times 10^{-6}(\mathrm{pb})$ & 2.64 & 1.2 & 0.15 & 11.3 \\
\hline$\Phi_{\mu}\left(k m^{-2} y r^{-1}\right)$ & 4 & 2.1 & 0.2 & 4 \\
\hline
\end{tabular}

Table 1. Table of benchmark points allowed by WMAP/PLANCK data of eq. (3.1) as well as direct detection bounds from the LUX and indirect detection constraints from the IceCube. All the benchmark points are for $\tan \beta=10$. BP1 (BP3) and BP2 (BP4) correspond to the case of a higgsino (wino)-LSP undergoing slepton and squark coannihilations respectively. The relevant SM parameters used are $m_{t}^{\text {pole }}=173.2 \mathrm{GeV}, m_{b}^{\overline{M S}}=4.19 \mathrm{GeV}$ and $m_{\tau}=1.77 \mathrm{GeV}$. 


\section{Conclusion}

A bino-dominated LSP generally produces overabundant DM. A bino-like LSP relies mostly on the bulk-annihilation or t-channel slepton exchange mechanism, a disfavored scenario in the context of LHC data. A bino can also be a DM candidate with the help of coannihilations with sleptons (typically staus), or coannihilations with suitable electroweakinos as in the Focus Point/Hyperbolic Branch region, or it can take the help of s-channel Higgs mediation for pair annihilation in order to satisfy the DM relic density limits. On the other hand, in MSSM there are theoretical as well as phenomenological motivations to study higgsino and wino-dominated LSPs. When the LSP turns out to be a higgsino, these processes include pair-annihilation and coannihilations among $\widetilde{\chi}_{1}^{0}, \widetilde{\chi}_{1}^{ \pm}$and $\widetilde{\chi}_{2}^{0}$. The same for a wino-LSP situation include coannihilations between $\widetilde{\chi}_{1}^{0}$ and $\widetilde{\chi}_{1}^{ \pm}$. It is known that these processes are too strong to cause the LSP to become an underabundant component of DM unless its mass is around $1 \mathrm{TeV}$ for a higgsino or a little above $2 \mathrm{TeV}$ for a wino type of LSP. We consider a compressed scenario of pMSSM where sfermions may take a very significant role as coannihilating sparticles. Our purpose is to examine how light the LSP as a higgsino or a wino can be while it satisfies both the lower and the upper limit of the DM relic density as given by the WMAP/PLANCK data. We choose two representative values of $\tan \beta$, namely 10 and 30 and consider both sleptons and squarks as coannihilating partners. In regard to the LSP-slepton coannihilations we consider all the three generations of sleptons including also the sneutrinos while keeping the squark masses heavy. We perform the analysis by requiring a maximum of $20 \%$ mass difference between that of the LSP and each of its coannihilating partners. Consideration of the slepton coannihilations reduces the effective cross section leading to an increase in the relic density. This is how the relic density gets modified or in other words this is how the lower limit of the LSP mass satisfying the relic density limits decreases. We find that for a higgsino dominated LSP the lowest LSP mass that satisfies the relic density limits is about $450 \mathrm{GeV}$, about a $60 \%$ reduction corresponding to the case of no sfermion coannihilations and this occurs for $\tan \beta=10$. The dependence on $\tan \beta$ comes via the $\mathrm{L}-\mathrm{R}$ mixing of tau-sleptons and the exponential suppression generically associated with coannihilation toward the effective annihilation cross-section. The same reduction in the lower limit for a wino type of LSP occurs for $\tan \beta=10$ and it is about $1.1 \mathrm{TeV}$, more than a 100 percent reduction in the value corresponding to the case of no sfermion coannihilations. For squarks, we allowed coannihilations with only the first two generations of squarks while imposing a similar $20 \%$ limit as before for the deviation of masses of the coannihilating particles from the LSP mass keeping the third generation of squarks as well as sleptons of all the generations very heavy. The reason of omitting the third generation lies in the fact that a large splitting between the two top-squark masses as required by a $125 \mathrm{GeV}$ Higgs boson would take away a lot of parameter space if we need a uniform $20 \%$ limit for the difference of each of the squarks and the LSP masses. In the absence of coannihilating third generation of squarks, our results become essentially independent of $\tan \beta$. The lowest LSP mass satisfying the relic density limits is about $840 \mathrm{GeV}$ for the higgsino case, only a modest reduction by 10-15\% from the generic higgsino LSP scenario. For the higgsino-squark coannihilation 
scenario we additionally obtain a region of parameter space where the relic density is decreased when squark coannihilations come to the picture, thus increasing the upper limit of the LSP mass satisfying the relic density data. This happens only in a very limited zone of parameter space with nearly degenerate squark and LSP masses and toward the end of the upper limit of the LSP mass satisfying the DM relic density data. Coming to wino, the lower limit of the LSP mass with the above squark coannihilations is around $1.1 \mathrm{TeV}$. Additionally, computation for a scenario of combined slepton and squark coannihilations shows that the lower limit of higgsino-LSP is about $500 \mathrm{GeV}$ whereas for a wino-LSP the same is about $900 \mathrm{GeV}$. We also note that throughout our study we consider the CP-odd Higgs boson $(A)$ to be sufficiently heavy so as to avoid an s-channel $A$-pole.

We further analyze the direct and indirect detection prospects of DM for the above types of LSPs for the two kinds of sfermion coannihilations considered in this work. In the part of the analysis that involves squark-LSP coannihilations, because of the near degeneracy of squarks with the LSP, the squark exchange diagrams in the direct detection cross section can be very important. These may even exceed the contributions from the Higgs exchange diagrams which typically dominate the generic MSSM parameter space.

The SI direct detection cross section may exceed the recent LUX data for a higgsino type of LSP undergoing slepton coannihilations for a mass below $600 \mathrm{GeV}$. For squark coannihilations, the above number is about $840 \mathrm{GeV}$. The corresponding number for the case of slepton plus squark coannihilations is around $680 \mathrm{GeV}$. The same occurs at around $1.27 \mathrm{TeV}$ for a wino-LSP undergoing squark coannihilations whereas there is no direct detection constraint for the part of the study involving slepton coannihilations. The case of combined slepton and squark coannihilations gives a lower mass limit of a wino DM as $1 \mathrm{TeV}$. However, in spite of the appearance of the above limits we must keep in mind that there can be an order of magnitude of uncertainty in the computation of the SI direct detection cross-section. This may potentially lower the above mass limits by 10 to $15 \%$. The indirect detection data such as that from the IceCube for the muon flux do not put any additional constraint than whatever is given by the relic density and the SI direct detection cross section data in combination. Regarding future experiments, XENON1T would be able to probe only the higgsino LSP scenario with both kinds of sfermion coannihilations. Finally, with relevant bounds from ATLAS and CMS being satisfied, we pointed out that for LSP and $\widetilde{\chi}_{1}^{ \pm}$either being a higgsino or a wino dominated in nature there is hardly any collider bound to worry about while considering the compressed pMSSM scenario where the sfermion masses would be suitable for DM coannihilations.

\section{Acknowledgments}

$\mathrm{UC}$ is thankful to receive the hospitality from CERN Theory division where a major part of this work was completed. MC would like to thank TRR33 "The Dark Universe" project for financial support.

Open Access. This article is distributed under the terms of the Creative Commons Attribution License (CC-BY 4.0), which permits any use, distribution and reproduction in any medium, provided the original author(s) and source are credited. 


\section{References}

[1] H.P. Nilles, Supersymmetry, Supergravity and Particle Physics, Phys. Rept. 110 (1984) 1 [INSPIRE].

[2] J.D. Lykken, Introduction to supersymmetry, hep-th/9612114 [INSPIRE].

[3] J. Wess and J. Bagger, Supersymmetry and Supergravity, second edition, Princeton (1991).

[4] H.E. Haber and G.L. Kane, The Search for Supersymmetry: Probing Physics Beyond the Standard Model, Phys. Rept. 117 (1985) 75 [INSPIRE].

[5] S.P. Martin, A Supersymmetry primer, Adv. Ser. Direct. High Energy Phys. 21 (2010) 1 [hep-ph/9709356] [INSPIRE].

[6] D.J.H. Chung, L.L. Everett, G.L. Kane, S.F. King, J.D. Lykken and L.-T. Wang, The Soft supersymmetry breaking Lagrangian: Theory and applications, Phys. Rept. 407 (2005) 1 [hep-ph/0312378] [INSPIRE].

[7] M. Drees, P. Roy and R.M. Godbole, Theory and Phenomenology of Sparticles, World Scientific, Singapore (2005).

[8] H. Baer and X. Tata, Weak scale supersymmetry: From superfields to scattering events, Cambridge University Press, Cambridge U.K. (2006).

[9] G. Jungman, M. Kamionkowski and K. Griest, Supersymmetric dark matter, Phys. Rept. 267 (1996) 195 [hep-ph/9506380] [INSPIRE].

[10] G. Bertone, D. Hooper and J. Silk, Particle dark matter: Evidence, candidates and constraints, Phys. Rept. 405 (2005) 279 [hep-ph/0404175] [INSPIRE].

[11] A.H. Chamseddine, R.L. Arnowitt and P. Nath, Locally Supersymmetric Grand Unification, Phys. Rev. Lett. 49 (1982) 970 [INSPIRE].

[12] R. Barbieri, S. Ferrara and C.A. Savoy, Gauge Models with Spontaneously Broken Local Supersymmetry, Phys. Lett. B 119 (1982) 343 [inSPIRE].

[13] L.J. Hall, J.D. Lykken and S. Weinberg, Supergravity as the Messenger of Supersymmetry Breaking, Phys. Rev. D 27 (1983) 2359 [INSPIRE].

[14] P. Nath, R.L. Arnowitt and A.H. Chamseddine, Gauge Hierarchy in Supergravity Guts, Nucl. Phys. B 227 (1983) 121 [inSPIRE].

[15] N. Ohta, Grand unified theories based on local supersymmetry, Prog. Theor. Phys. 70 (1983) 542 [INSPIRE].

[16] P. Nath, R. Arnowitt and A.H. Chamseddine, Applied $N=1$ Supergravity, World Scientific, Singapore (1984).

[17] ATLAS collaboration, Observation of a new particle in the search for the Standard Model Higgs boson with the ATLAS detector at the LHC, Phys. Lett. B 716 (2012) 1 [arXiv:1207.7214] [INSPIRE].

[18] CMS collaboration, Observation of a new boson at a mass of $125 \mathrm{GeV}$ with the CMS experiment at the LHC, Phys. Lett. B 716 (2012) 30 [arXiv:1207.7235] [INSPIRE].

[19] ATLAS, CMS collaborations, Combined Measurement of the Higgs Boson Mass in pp Collisions at $\sqrt{s}=7$ and 8 TeV with the ATLAS and CMS Experiments, Phys. Rev. Lett. 114 (2015) 191803 [arXiv: 1503.07589] [INSPIRE]. 
[20] M. Citron, J. Ellis, F. Luo, J. Marrouche, K.A. Olive and K.J. de Vries, End of the CMSSM coannihilation strip is nigh, Phys. Rev. D 87 (2013) 036012 [arXiv:1212.2886] [INSPIRE].

[21] K. Kowalska, L. Roszkowski and E.M. Sessolo, Two ultimate tests of constrained supersymmetry, JHEP 06 (2013) 078 [arXiv: 1302.5956] [INSPIRE].

[22] S. Henrot-Versillé, R. Lafaye, T. Plehn, M. Rauch, D. Zerwas, S. Plaszczynski et al., Constraining Supersymmetry using the relic density and the Higgs boson, Phys. Rev. D 89 (2014) 055017 [arXiv: 1309.6958] [INSPIRE].

[23] P. Bechtle et al., Constrained Supersymmetry after the Higgs Boson Discovery: A global analysis with Fittino, PoS(EPS-HEP 2013) 313 [arXiv:1310.3045] [INSPIRE].

[24] J. Ellis, Supersymmetric Fits after the Higgs Discovery and Implications for Model Building, Eur. Phys. J. C 74 (2014) 2732 [arXiv:1312.5426] [INSPIRE].

[25] L. Roszkowski, E.M. Sessolo and A.J. Williams, What next for the CMSSM and the NUHM: Improved prospects for superpartner and dark matter detection, JHEP 08 (2014) 067 [arXiv: 1405.4289] [INSPIRE].

[26] K.L. Chan, U. Chattopadhyay and P. Nath, Naturalness, weak scale supersymmetry and the prospect for the observation of supersymmetry at the Tevatron and at the CERN LHC, Phys. Rev. D 58 (1998) 096004 [hep-ph/9710473] [INSPIRE].

[27] U. Chattopadhyay, A. Corsetti and P. Nath, WMAP constraints, SUSY dark matter and implications for the direct detection of SUSY, Phys. Rev. D 68 (2003) 035005 [hep-ph/0303201] [INSPIRE].

[28] S. Akula, M. Liu, P. Nath and G. Peim, Naturalness, Supersymmetry and Implications for LHC and Dark Matter, Phys. Lett. B 709 (2012) 192 [arXiv:1111.4589] [INSPIRE].

[29] J.L. Feng, K.T. Matchev and T. Moroi, Focus points and naturalness in supersymmetry, Phys. Rev. D 61 (2000) 075005 [hep-ph/9909334] [INSPIRE].

[30] J.L. Feng, K.T. Matchev and T. Moroi, Multi - TeV scalars are natural in minimal supergravity, Phys. Rev. Lett. 84 (2000) 2322 [hep-ph/9908309] [INSPIRE].

[31] J.L. Feng, K.T. Matchev and F. Wilczek, Neutralino dark matter in focus point supersymmetry, Phys. Lett. B 482 (2000) 388 [hep-ph/0004043] [INSPIRE].

[32] U. Chattopadhyay, A. Datta, A. Datta, A. Datta and D.P. Roy, LHC signature of the minimal SUGRA model with a large soft scalar mass, Phys. Lett. B 493 (2000) 127 [hep-ph/0008228] [INSPIRE].

[33] U. Chattopadhyay, T. Ibrahim and D.P. Roy, Electron and neutron electric dipole moments in the focus point scenario of SUGRA model, Phys. Rev. D 64 (2001) 013004 [hep-ph/0012337] [INSPIRE].

[34] J.L. Feng and F. Wilczek, Advantages and distinguishing features of focus point supersymmetry, Phys. Lett. B 631 (2005) 170 [hep-ph/0507032] [INSPIRE].

[35] S.P. Das, A. Datta, M. Guchait, M. Maity and S. Mukherjee, Focus Point SUSY at the LHC Revisited, Eur. Phys. J. C 54 (2008) 645 [arXiv:0708.2048] [INSPIRE].

[36] H. Baer, V. Barger and A. Mustafayev, Implications of a $125 \mathrm{GeV}$ Higgs scalar for LHC SUSY and neutralino dark matter searches, Phys. Rev. D 85 (2012) 075010 [arXiv:1112.3017] [INSPIRE]. 
[37] J. Ellis and K.A. Olive, Revisiting the Higgs Mass and Dark Matter in the CMSSM, Eur. Phys. J. C 72 (2012) 2005 [arXiv: 1202.3262] [INSPIRE].

[38] O. Buchmueller et al., The CMSSM and NUHM1 in Light of 7 TeV LHC, $B_{s} \rightarrow \mu^{+} \mu^{-}$and XENON100 Data, Eur. Phys. J. C 72 (2012) 2243 [arXiv:1207.7315] [InSPIRE].

[39] O. Buchmueller et al., The CMSSM and NUHM1 after LHC Run 1, Eur. Phys. J. C 74 (2014) 2922 [arXiv: 1312.5250] [INSPIRE].

[40] J.R. Ellis, K. Enqvist, D.V. Nanopoulos and K. Tamvakis, Gaugino Masses and Grand Unification, Phys. Lett. B 155 (1985) 381 [INSPIRE].

[41] M. Drees, Phenomenological Consequences of $N=1$ Supergravity Theories With Nonminimal Kinetic Energy Terms for Vector Superfields, Phys. Lett. B 158 (1985) 409 [INSPIRE].

[42] A. Corsetti and P. Nath, Gaugino mass nonuniversality and dark matter in SUGRA, strings and D-brane models, Phys. Rev. D 64 (2001) 125010 [hep-ph/0003186] [INSPIRE].

[43] U. Chattopadhyay and P. Nath, $b-\tau$ unification, $g_{\mu}-2$, the $\vec{b} s+\gamma$ constraint and nonuniversalities, Phys. Rev. D 65 (2002) 075009 [hep-ph/0110341] [INSPIRE].

[44] G. Anderson, C.H. Chen, J.F. Gunion, J.D. Lykken, T. Moroi and Y. Yamada, Motivations for and implications of nonuniversal GUT scale boundary conditions for soft SUSY breaking parameters, eConf C 960625 (1996) SUP107 [hep-ph/9609457] [INSPIRE].

[45] K. Huitu, Y. Kawamura, T. Kobayashi and K. Puolamaki, Phenomenological constraints on SUSY SU(5) GUTs with nonuniversal gaugino masses, Phys. Rev. D 61 (2000) 035001 [hep-ph/9903528] [INSPIRE].

[46] G. Anderson, H. Baer, C.-h. Chen and X. Tata, The Reach of Fermilab Tevatron upgrades for SU(5) supergravity models with nonuniversal gaugino masses, Phys. Rev. D 61 (2000) 095005 [hep-ph/9903370] [INSPIRE].

[47] J. Chakrabortty and A. Raychaudhuri, A Note on dimension-5 operators in GUTs and their impact, Phys. Lett. B 673 (2009) 57 [arXiv:0812.2783] [INSPIRE].

[48] S.P. Martin, Non-universal gaugino masses from non-singlet F-terms in non-minimal unified models, Phys. Rev. D 79 (2009) 095019 [arXiv:0903.3568] [InSPIRE].

[49] U. Chattopadhyay and D.P. Roy, Higgsino dark matter in a SUGRA model with nonuniversal gaugino masses, Phys. Rev. D 68 (2003) 033010 [hep-ph/0304108] [INSPIRE].

[50] U. Chattopadhyay, A. Corsetti and P. Nath, Supersymmetric dark matter and Yukawa unification, Phys. Rev. D 66 (2002) 035003 [hep-ph/0201001] [INSPIRE].

[51] U. Chattopadhyay, D. Choudhury and D. Das, Large evolution of the bilinear Higgs coupling parameter in SUSY models and reduction of phase sensitivity, Phys. Rev. D 72 (2005) 095015 [hep-ph/0509228] [INSPIRE].

[52] K. Huitu, J. Laamanen, P.N. Pandita and S. Roy, Phenomenology of non-universal gaugino masses in supersymmetric grand unified theories, Phys. Rev. D 72 (2005) 055013 [hep-ph/0502100] [INSPIRE].

[53] G. Bélanger, F. Boudjema, A. Cottrant, A. Pukhov and A. Semenov, WMAP constraints on SUGRA models with non-universal gaugino masses and prospects for direct detection, Nucl. Phys. B 706 (2005) 411 [hep-ph/0407218] [INSPIRE]. 
[54] S.F. King, J.P. Roberts and D.P. Roy, Natural dark matter in SUSY GUTs with non-universal gaugino masses, JHEP 10 (2007) 106 [arXiv:0705.4219] [INSPIRE].

[55] S. Bhattacharya, A. Datta and B. Mukhopadhyaya, Non-universal gaugino masses: A Signal-based analysis for the Large Hadron Collider, JHEP 10 (2007) 080 [arXiv:0708.2427] [INSPIRE].

[56] K. Huitu, R. Kinnunen, J. Laamanen, S. Lehti, S. Roy and T. Salminen, Search for Higgs Bosons in SUSY Cascades in CMS and Dark Matter with Non-universal Gaugino Masses, Eur. Phys. J. C 58 (2008) 591 [arXiv:0808.3094] [inSPIRE].

[57] S. Bhattacharya and J. Chakrabortty, Gaugino mass non-universality in an $\mathrm{SO}(10)$ supersymmetric Grand Unified Theory: Low-energy spectra and collider signals, Phys. Rev. D 81 (2010) 015007 [arXiv:0903.4196] [INSPIRE].

[58] U. Chattopadhyay, D. Das and D.P. Roy, Mixed Neutralino Dark Matter in Nonuniversal Gaugino Mass Models, Phys. Rev. D 79 (2009) 095013 [arXiv:0902.4568] [INSPIRE].

[59] M. Guchait, D.P. Roy and D. Sengupta, Probing a Mixed Neutralino Dark Matter Model at the $7 \mathrm{TeV}$ LHC, Phys. Rev. D 85 (2012) 035024 [arXiv:1109.6529] [InSPIRE].

[60] S. Mohanty, S. Rao and D.P. Roy, Predictions of a Natural SUSY Dark Matter Model for Direct and Indirect Detection Experiments, JHEP 11 (2012) 175 [arXiv:1208.0894] [INSPIRE].

[61] S. Mohanty, S. Rao and D.P. Roy, Reconciling the muon $g-2$ and dark matter relic density with the LHC results in nonuniversal gaugino mass models, JHEP 09 (2013) 027 [arXiv:1303.5830] [INSPIRE].

[62] J. Chakrabortty, S. Mohanty and S. Rao, Non-universal gaugino mass GUT models in the light of dark matter and LHC constraints, JHEP 02 (2014) 074 [arXiv:1310.3620] [INSPIRE].

[63] S.P. Martin, Nonuniversal gaugino masses and seminatural supersymmetry in view of the Higgs boson discovery, Phys. Rev. D 89 (2014) 035011 [arXiv:1312.0582] [INSPIRE].

[64] S.P. Das, M. Guchait and D.P. Roy, Testing SUSY models for the muon g-2 anomaly via chargino-neutralino pair production at the LHC, Phys. Rev. D 90 (2014) 055011 [arXiv:1406.6925] [INSPIRE].

[65] I. Gogoladze, F. Nasir, Q. Shafi and C.S. Un, Nonuniversal Gaugino Masses and Muon g-2, Phys. Rev. D 90 (2014) 035008 [arXiv: 1403.2337] [INSPIRE].

[66] U. Chattopadhyay, D. Choudhury, M. Drees, P. Konar and D.P. Roy, Looking for a heavy Higgsino LSP in collider and dark matter experiments, Phys. Lett. B 632 (2006) 114 [hep-ph/0508098] [INSPIRE].

[67] M. Chakraborti, U. Chattopadhyay, S. Rao and D.P. Roy, Higgsino Dark Matter in Nonuniversal Gaugino Mass Models, Phys. Rev. D 91 (2015) 035022 [arXiv:1411.4517] [INSPIRE].

[68] L. Roszkowski, R. Ruiz de Austri, R. Trotta, Y.-L.S. Tsai and T.A. Varley, Global fits of the Non-Universal Higgs Model, Phys. Rev. D 83 (2011) 015014 [Erratum ibid. D 83 (2011) 039901] [arXiv: 0903.1279] [INSPIRE].

[69] H. Baer, A. Mustafayev, S. Profumo, A. Belyaev and X. Tata, Direct, indirect and collider detection of neutralino dark matter in SUSY models with non-universal Higgs masses, JHEP 07 (2005) 065 [hep-ph/0504001] [INSPIRE]. 
[70] J.R. Ellis, K.A. Olive and Y. Santoso, The MSSM parameter space with nonuniversal Higgs masses, Phys. Lett. B 539 (2002) 107 [hep-ph/0204192] [InSPIRE].

[71] G.G. Ross, K. Schmidt-Hoberg and F. Staub, On the MSSM Higgsino mass and fine tuning, Phys. Lett. B 759 (2016) 110 [arXiv:1603.09347] [InSPIRE].

[72] U. Chattopadhyay and A. Dey, Probing Non-holomorphic MSSM via precision constraints, dark matter and LHC data, JHEP 10 (2016) 027 [arXiv:1604.06367] [INSPIRE].

[73] N. Arkani-Hamed, A. Delgado and G.F. Giudice, The Well-tempered neutralino, Nucl. Phys. B 741 (2006) 108 [hep-ph/0601041] [INSPIRE].

[74] G.F. Giudice, M.A. Luty, H. Murayama and R. Rattazzi, Gaugino mass without singlets, JHEP 12 (1998) 027 [hep-ph/9810442] [INSPIRE].

[75] L. Randall and R. Sundrum, Out of this world supersymmetry breaking, Nucl. Phys. B 557 (1999) 79 [hep-th/9810155] [INSPIRE].

[76] J.A. Bagger, T. Moroi and E. Poppitz, Anomaly mediation in supergravity theories, JHEP 04 (2000) 009 [hep-th/9911029] [INSPIRE].

[77] U. Chattopadhyay, D.K. Ghosh and S. Roy, Constraining anomaly mediated supersymmetry breaking framework via on going muon g-2 experiment at Brookhaven, Phys. Rev. D 62 (2000) 115001 [hep-ph/0006049] [INSPIRE].

[78] U. Chattopadhyay, D. Das, P. Konar and D.P. Roy, Looking for a heavy wino LSP in collider and dark matter experiments, Phys. Rev. D 75 (2007) 073014 [hep-ph/0610077] [INSPIRE].

[79] J. Hisano, S. Matsumoto, M. Nagai, O. Saito and M. Senami, Non-perturbative effect on thermal relic abundance of dark matter, Phys. Lett. B 646 (2007) 34 [hep-ph/0610249] [INSPIRE].

[80] B. Bhattacherjee, M. Ibe, K. Ichikawa, S. Matsumoto and K. Nishiyama, Wino Dark Matter and Future dSph Observations, JHEP 07 (2014) 080 [arXiv: 1405.4914] [INSPIRE].

[81] M. Beneke, A. Bharucha, F. Dighera, C. Hellmann, A. Hryczuk, S. Recksiegel et al., Relic density of wino-like dark matter in the MSSM, JHEP 03 (2016) 119 [arXiv:1601.04718] [INSPIRE].

[82] M. Cirelli, A. Strumia and M. Tamburini, Cosmology and Astrophysics of Minimal Dark Matter, Nucl. Phys. B 787 (2007) 152 [arXiv:0706.4071] [InSPIRE].

[83] A. Masiero, S. Profumo and P. Ullio, Neutralino dark matter detection in split supersymmetry scenarios, Nucl. Phys. B 712 (2005) 86 [hep-ph/0412058] [INSPIRE].

[84] T. Cohen, M. Lisanti, A. Pierce and T.R. Slatyer, Wino Dark Matter Under Siege, JCAP 10 (2013) 061 [arXiv:1307.4082] [INSPIRE].

[85] M. Baumgart, I.Z. Rothstein and V. Vaidya, Constraints on Galactic Wino Densities from Gamma Ray Lines, JHEP 04 (2015) 106 [arXiv: 1412.8698] [INSPIRE].

[86] H. Baer, V. Barger, P. Huang, D. Mickelson, M. Padeffke-Kirkland and X. Tata, Natural SUSY with a bino- or wino-like LSP, Phys. Rev. D 91 (2015) 075005 [arXiv:1501.06357] [INSPIRE].

[87] M. Ibe, S. Matsumoto, S. Shirai and T.T. Yanagida, Wino Dark Matter in light of the AMS-02 2015 Data, Phys. Rev. D 91 (2015) 111701 [arXiv:1504.05554] [INSPIRE]. 
[88] A. Hryczuk, I. Cholis, R. Iengo, M. Tavakoli and P. Ullio, Indirect Detection Analysis: Wino Dark Matter Case Study, JCAP 07 (2014) 031 [arXiv:1401.6212] [INSPIRE].

[89] ATLAS collaboration, Search for supersymmetry at $\sqrt{s}=8$ TeV in final states with jets and two same-sign leptons or three leptons with the ATLAS detector, JHEP 06 (2014) 035 [arXiv: 1404.2500] [INSPIRE].

[90] ATLAS collaboration, Search for squarks and gluinos with the ATLAS detector in final states with jets and missing transverse momentum using $\sqrt{s}=8$ TeV proton-proton collision data, JHEP 09 (2014) 176 [arXiv:1405.7875] [INSPIRE].

[91] ATLAS collaboration, Search for strong production of supersymmetric particles in final states with missing transverse momentum and at least three b-jets at $\sqrt{s}=8 \mathrm{TeV}$ proton-proton collisions with the ATLAS detector, JHEP 10 (2014) 024 [arXiv:1407.0600] [INSPIRE].

[92] ATLAS collaboration, Search for squarks and gluinos in events with isolated leptons, jets and missing transverse momentum at $\sqrt{s}=8 \mathrm{TeV}$ with the ATLAS detector, JHEP 04 (2015) 116 [arXiv:1501.03555] [INSPIRE].

[93] ATLAS collaboration, Search for supersymmetry in events containing a same-flavour opposite-sign dilepton pair, jets and large missing transverse momentum in $\sqrt{s}=8 \mathrm{TeV} \mathrm{pp}$ collisions with the ATLAS detector, Eur. Phys. J. C 75 (2015) 318 [Erratum ibid. C 75 (2015) 463] [arXiv: 1503.03290] [INSPIRE].

[94] CMS collaboration, Search for supersymmetry in pp collisions at $\sqrt{s}=8 \mathrm{TeV}$ in events with a single lepton, large jet multiplicity and multiple b jets, Phys. Lett. B 733 (2014) 328 [arXiv:1311.4937] [INSPIRE].

[95] CMS collaboration, Search for new physics in events with same-sign dileptons and jets in pp collisions at $\sqrt{s}=8 \mathrm{TeV}$, JHEP 01 (2014) 163 [Erratum ibid. 1501 (2015) 014] [arXiv:1311.6736] [INSPIRE].

[96] CMS collaboration, Search for new physics in the multijet and missing transverse momentum final state in proton-proton collisions at $\sqrt{s}=8 \mathrm{TeV}$, JHEP 06 (2014) 055 [arXiv:1402.4770] [INSPIRE].

[97] CMS collaboration, Searches for supersymmetry based on events with $b$ jets and four $W$ bosons in pp collisions at $8 \mathrm{TeV}$, Phys. Lett. B $\mathbf{7 4 5}$ (2015) 5 [arXiv:1412.4109] [InSPIRE].

[98] CMS collaboration, Search for Supersymmetry Using Razor Variables in Events with $b$-Tagged Jets in pp Collisions at $\sqrt{s}=8$ TeV, Phys. Rev. D 91 (2015) 052018 [arXiv: 1502.00300] [INSPIRE].

[99] CMS collaboration, Searches for Supersymmetry using the $M_{T 2}$ Variable in Hadronic Events Produced in pp Collisions at 8 TeV, JHEP 05 (2015) 078 [arXiv:1502.04358] [INSPIRE].

[100] CMS collaboration, Search for Physics Beyond the Standard Model in Events with Two Leptons, Jets and Missing Transverse Momentum in pp Collisions at $\sqrt{s}=8 \mathrm{TeV}$, JHEP 04 (2015) 124 [arXiv: 1502.06031] [INSPIRE].

[101] ATLAS collaboration, Search for direct production of charginos and neutralinos in events with three leptons and missing transverse momentum in $\sqrt{s}=8 T e V$ pp collisions with the ATLAS detector, JHEP 04 (2014) 169 [arXiv: 1402.7029] [INSPIRE]. 
[102] ATLAS collaboration, Search for direct production of charginos, neutralinos and sleptons in final states with two leptons and missing transverse momentum in pp collisions at $\sqrt{s}=$ $8 \mathrm{TeV}$ with the ATLAS detector, JHEP 05 (2014) 071 [arXiv:1403.5294] [INSPIRE].

[103] ATLAS collaboration, Search for the direct production of charginos, neutralinos and staus in final states with at least two hadronically decaying taus and missing transverse momentum in pp collisions at $\sqrt{s}=8$ TeV with the ATLAS detector, JHEP 10 (2014) 096 [arXiv: 1407.0350] [INSPIRE].

[104] ATLAS collaboration, Search for direct pair production of a chargino and a neutralino decaying to the $125 \mathrm{GeV}$ Higgs boson in $\sqrt{s}=8 \mathrm{TeV}$ pp collisions with the ATLAS detector, Eur. Phys. J. C 75 (2015) 208 [arXiv:1501.07110] [INSPIRE].

[105] ATLAS collaboration, Search for electroweak production of supersymmetric particles in final states with tau leptons in $\sqrt{s}=13 T e V$ pp collisions with the ATLAS detector, ATLAS-CONF-2016-093 (2016).

[106] ATLAS collaboration, Search for supersymmetry with two and three leptons and missing transverse momentum in the final state at $\sqrt{s}=13 \mathrm{TeV}$ with the ATLAS detector, ATLAS-CONF-2016-096 (2016).

[107] CMS collaboration, Searches for electroweak production of charginos, neutralinos and sleptons decaying to leptons and $W, Z$ and Higgs bosons in pp collisions at $8 \mathrm{TeV}$, Eur. Phys. J. C 74 (2014) 3036 [arXiv:1405.7570] [inSPIRE].

[108] CMS collaboration, Searches for electroweak neutralino and chargino production in channels with Higgs, $Z$ and $W$ bosons in pp collisions at 8 TeV, Phys. Rev. D 90 (2014) 092007 [arXiv: 1409.3168] [INSPIRE].

[109] CMS collaboration, Search for electroweak SUSY production in multilepton final states in pp collisions at $\sqrt{s}=13 \mathrm{TeV}$ with 12.9/fb, CMS-PAS-SUS-16-024 (2016).

[110] CMS collaboration, Search for electroweak production of charginos in final states with two tau leptons in pp collisions at $\sqrt{s}=8 \mathrm{TeV}$, CMS-PAS-SUS-14-022.

[111] CMS collaboration, Search for electroweak production of charginos and neutralinos in the WH final state at $13 \mathrm{TeV}$, CMS-PAS-SUS-16-026 (2016).

[112] ATLAS collaboration, Search for direct production of charginos and neutralinos in events with three leptons and missing transverse momentum in $21 \mathrm{fb}^{-1}$ of $p p$ collisions at $\sqrt{s}=8 \mathrm{TeV}$ with the ATLAS detector, ATLAS-CONF-2013-035 (2013).

[113] M. Chakraborti, U. Chattopadhyay, A. Choudhury, A. Datta and S. Poddar, The Electroweak Sector of the pMSSM in the Light of LHC - $8 \mathrm{TeV}$ and Other Data, JHEP 07 (2014) 019 [arXiv: 1404.4841] [inSPIRE].

[114] D. Ghosh, M. Guchait and D. Sengupta, Higgs Signal in Chargino-Neutralino Production at the LHC, Eur. Phys. J. C 72 (2012) 2141 [arXiv:1202.4937] [INSPIRE].

[115] K. Howe and P. Saraswat, Excess Higgs Production in Neutralino Decays, JHEP 10 (2012) 065 [arXiv: 1208.1542] [INSPIRE].

[116] A. Arbey, M. Battaglia and F. Mahmoudi, Higgs Production in Neutralino Decays in the MSSM - The LHC and a Future $e^{+} e^{-}$Collider, Eur. Phys. J. C 75 (2015) 108 [arXiv: 1212.6865] [INSPIRE].

[117] A. Choudhury and A. Datta, Neutralino dark matter confronted by the LHC constraints on Electroweak SUSY signals, JHEP 09 (2013) 119 [arXiv: 1305.0928] [INSPIRE]. 
[118] A. Bharucha, S. Heinemeyer and F. von der Pahlen, Direct Chargino-Neutralino Production at the LHC: Interpreting the Exclusion Limits in the Complex MSSM, Eur. Phys. J. C 73 (2013) 2629 [arXiv: 1307.4237] [INSPIRE].

[119] T. Han, S. Padhi and S. Su, Electroweakinos in the Light of the Higgs Boson, Phys. Rev. D 88 (2013) 115010 [arXiv: 1309.5966] [INSPIRE].

[120] A. Papaefstathiou, K. Sakurai and M. Takeuchi, Higgs boson to di-tau channel in Chargino-Neutralino searches at the LHC, JHEP 08 (2014) 176 [arXiv:1404.1077] [INSPIRE].

[121] F. Yu, Anatomizing Exotic Production of the Higgs Boson, Phys. Rev. D 90 (2014) 015009 [arXiv: 1404.2924] [INSPIRE].

[122] J. Eckel, M.J. Ramsey-Musolf, W. Shepherd and S. Su, Impact of LSP Character on Slepton Reach at the LHC, JHEP 11 (2014) 117 [arXiv:1408.2841] [INSPIRE].

[123] C. Han, L. Wu, J.M. Yang, M. Zhang and Y. Zhang, New approach for detecting a compressed bino/wino at the LHC, Phys. Rev. D 91 (2015) 055030 [arXiv:1409.4533] [INSPIRE].

[124] C. Han, Probing light bino and higgsinos at the LHC, arXiv:1409.7000 [INSPIRE].

[125] J. Bramante, P.J. Fox, A. Martin, B. Ostdiek, T. Plehn, T. Schell et al., Relic Neutralino Surface at a $100 \mathrm{TeV}$ Collider, Phys. Rev. D 91 (2015) 054015 [arXiv:1412.4789] [INSPIRE].

[126] A. Choudhury, K. Kowalska, L. Roszkowski, E.M. Sessolo and A.J. Williams, Less-simplified models of dark matter for direct detection and the LHC, JHEP 04 (2016) 182 [arXiv: 1509.05771] [INSPIRE].

[127] J. Bramante, N. Desai, P. Fox, A. Martin, B. Ostdiek and T. Plehn, Towards the Final Word on Neutralino Dark Matter, Phys. Rev. D 93 (2016) 063525 [arXiv:1510.03460] [INSPIRE].

[128] K. Hamaguchi and K. Ishikawa, Prospects for Higgs- and Z-resonant Neutralino Dark Matter, Phys. Rev. D 93 (2016) 055009 [arXiv: 1510.05378] [INSPIRE].

[129] J. Cao, Y. He, L. Shang, W. Su and Y. Zhang, Testing the light dark matter scenario of the MSSM at the LHC, JHEP 03 (2016) 207 [arXiv: 1511.05386] [INSPIRE].

[130] A. Choudhury and S. Mondal, Revisiting the Exclusion Limits from Direct Chargino-Neutralino Production at the LHC, Phys. Rev. D 94 (2016) 055024 [arXiv: 1603. 05502] [INSPIRE].

[131] A. Datta, N. Ganguly and S. Poddar, New Limits on Heavier Electroweakinos and their LHC Signatures, Phys. Lett. B 763 (2016) 213 [arXiv:1606.04391] [INSPIRE].

[132] D. Chowdhury, K.M. Patel, X. Tata and S.K. Vempati, Indirect Searches of the Degenerate MSSM, Phys. Rev. D 95 (2017) 075025 [arXiv:1612.06471] [InSPIRE].

[133] M. Chakraborti, U. Chattopadhyay, A. Choudhury, A. Datta and S. Poddar, Reduced LHC constraints for higgsino-like heavier electroweakinos, JHEP 11 (2015) 050 [arXiv: 1507.01395] [INSPIRE].

[134] CMS collaboration, Search for dark matter, extra dimensions and unparticles in monojet events in proton-proton collisions at $\sqrt{s}=8 \mathrm{TeV}$, Eur. Phys. J. C $7 \mathbf{5}$ (2015) 235 [arXiv: 1408.3583] [INSPIRE]. 
[135] ATLAS collaboration, Search for new phenomena in final states with an energetic jet and large missing transverse momentum in pp collisions at $\sqrt{s}=8 \mathrm{TeV}$ with the ATLAS detector, Eur. Phys. J. C 75 (2015) 299 [arXiv: 1502.01518] [inSPIRE].

[136] D. Barducci, A. Belyaev, A.K.M. Bharucha, W. Porod and V. Sanz, Uncovering Natural Supersymmetry via the interplay between the LHC and Direct Dark Matter Detection, JHEP 07 (2015) 066 [arXiv: 1504.02472] [INSPIRE].

[137] MSSM Working Group collaboration, A. Djouadi et al., The Minimal supersymmetric standard model: Group summary report, hep-ph/9901246 [INSPIRE].

[138] K. Griest and D. Seckel, Three exceptions in the calculation of relic abundances, Phys. Rev. D 43 (1991) 3191 [INSPIRE].

[139] J.R. Ellis, T. Falk, K.A. Olive and M. Srednicki, Calculations of neutralino-stau coannihilation channels and the cosmologically relevant region of MSSM parameter space, Astropart. Phys. 13 (2000) 181 [Erratum ibid. 15 (2001) 413] [hep-ph/9905481] [INSPIRE].

[140] WMAP collaboration, G. Hinshaw et al., Nine-Year Wilkinson Microwave Anisotropy Probe (WMAP) Observations: Cosmological Parameter Results, Astrophys. J. Suppl. 208 (2013) 19 [arXiv:1212.5226] [inSPIRE].

[141] Planck collaboration, P.A.R. Ade et al., Planck 2013 results. XVI. Cosmological parameters, Astron. Astrophys. 571 (2014) A16 [arXiv:1303.5076] [inSPIRE].

[142] J. Edsjo, M. Schelke, P. Ullio and P. Gondolo, Accurate relic densities with neutralino, chargino and sfermion coannihilations in mSUGRA, JCAP 04 (2003) 001 [hep-ph/0301106] [INSPIRE].

[143] J. Edsjo and P. Gondolo, Neutralino relic density including coannihilations, Phys. Rev. D 56 (1997) 1879 [hep-ph/9704361] [INSPIRE].

[144] S. Profumo and A. Provenza, Increasing the neutralino relic abundance with slepton coannihilations: Consequences for indirect dark matter detection, JCAP 12 (2006) 019 [hep-ph/0609290] [INSPIRE].

[145] LUX collaboration, D.S. Akerib et al., Results from a search for dark matter in the complete LUX exposure, Phys. Rev. Lett. 118 (2017) 021303 [arXiv:1608. 07648] [INSPIRE].

[146] J. Hisano, S. Matsumoto, M.M. Nojiri and O. Saito, Direct detection of the Wino and Higgsino-like neutralino dark matters at one-loop level, Phys. Rev. D 71 (2005) 015007 [hep-ph/0407168] [INSPIRE].

[147] ICECuBE collaboration, R. Abbasi et al., Multi-year search for dark matter annihilations in the Sun with the AMANDA-II and IceCube detectors, Phys. Rev. D 85 (2012) 042002 [arXiv:1112.1840] [INSPIRE].

[148] IceCube collaboration, M.G. Aartsen et al., Search for dark matter annihilations in the Sun with the 79-string IceCube detector, Phys. Rev. Lett. 110 (2013) 131302 [arXiv: 1212.4097] [INSPIRE].

[149] J. Harz, B. Herrmann, M. Klasen, K. Kovarik and P. Steppeler, Theoretical uncertainty of the supersymmetric dark matter relic density from scheme and scale variations, Phys. Rev. D 93 (2016) 114023 [arXiv: 1602.08103] [INSPIRE].

[150] M. Klasen, K. Kovarik and P. Steppeler, SUSY-QCD corrections for direct detection of neutralino dark matter and correlations with relic density, Phys. Rev. D 94 (2016) 095002 [arXiv: 1607.06396] [INSPIRE]. 
[151] G. Bertone, F. Calore, S. Caron, R. Ruiz, J.S. Kim, R. Trotta et al., Global analysis of the pMSSM in light of the Fermi GeV excess: prospects for the LHC Run-II and astroparticle experiments, JCAP 04 (2016) 037 [arXiv:1507.07008] [INSPIRE].

[152] M. Badziak, M. Olechowski and P. Szczerbiak, Spin-dependent constraints on blind spots for thermal singlino-higgsino dark matter with(out) light singlets, JHEP 07 (2017) 050 [arXiv: 1705.00227] [INSPIRE].

[153] A. Djouadi, J.-L. Kneur and G. Moultaka, SuSpect: A Fortran code for the supersymmetric and Higgs particle spectrum in the MSSM, Comput. Phys. Commun. 176 (2007) 426 [hep-ph/0211331] [INSPIRE].

[154] G. Bélanger, F. Boudjema, A. Pukhov and A. Semenov, MicrOMEGAs $s_{3}$ : A program for calculating dark matter observables, Comput. Phys. Commun. 185 (2014) 960 [arXiv: 1305.0237] [INSPIRE].

[155] P. Gondolo, J. Edsjo, P. Ullio, L. Bergstrom, M. Schelke and E.A. Baltz, DarkSUSY: Computing supersymmetric dark matter properties numerically, JCAP 07 (2004) 008 [astro-ph/0406204] [INSPIRE].

[156] G. Degrassi, S. Heinemeyer, W. Hollik, P. Slavich and G. Weiglein, Towards high precision predictions for the MSSM Higgs sector, Eur. Phys. J. C 28 (2003) 133 [hep-ph/0212020] [INSPIRE].

[157] B.C. Allanach, A. Djouadi, J.L. Kneur, W. Porod and P. Slavich, Precise determination of the neutral Higgs boson masses in the MSSM, JHEP 09 (2004) 044 [hep-ph/0406166] [INSPIRE].

[158] S.P. Martin, Three-loop corrections to the lightest Higgs scalar boson mass in supersymmetry, Phys. Rev. D 75 (2007) 055005 [hep-ph/0701051] [INSPIRE].

[159] R.V. Harlander, P. Kant, L. Mihaila and M. Steinhauser, Higgs boson mass in supersymmetry to three loops, Phys. Rev. Lett. 100 (2008) 191602 [Erratum ibid. 101 (2008) 039901] [arXiv: 0803.0672] [INSPIRE].

[160] S. Heinemeyer, O. Stal and G. Weiglein, Interpreting the LHC Higgs Search Results in the MSSM, Phys. Lett. B 710 (2012) 201 [arXiv:1112.3026] [INSPIRE].

[161] A. Arbey, M. Battaglia, A. Djouadi and F. Mahmoudi, The Higgs sector of the phenomenological MSSM in the light of the Higgs boson discovery, JHEP 09 (2012) 107 [arXiv: 1207.1348] [INSPIRE].

[162] M. Drees, M.M. Nojiri, D.P. Roy and Y. Yamada, Light Higgsino dark matter, Phys. Rev. D 56 (1997) 276 [Erratum ibid. D 64 (2001) 039901] [hep-ph/9701219] [INSPIRE].

[163] G.D. Kribs, A. Martin and A. Menon, Natural Supersymmetry and Implications for Higgs physics, Phys. Rev. D 88 (2013) 035025 [arXiv: 1305.1313] [INSPIRE].

[164] G.F. Giudice and A. Pomarol, Mass degeneracy of the Higgsinos, Phys. Lett. B 372 (1996) 253 [hep-ph/9512337] [INSPIRE].

[165] H.-C. Cheng, B.A. Dobrescu and K.T. Matchev, Generic and chiral extensions of the supersymmetric standard model, Nucl. Phys. B 543 (1999) 47 [hep-ph/9811316] [inSPIRE].

[166] N.E. Bomark, A. Kvellestad, S. Lola, P. Osland and A.R. Raklev, Long lived charginos in Natural SUSY?, JHEP 05 (2014) 007 [arXiv: 1310.2788] [INSPIRE]. 
[167] T. Gherghetta, G.F. Giudice and J.D. Wells, Phenomenological consequences of supersymmetry with anomaly induced masses, Nucl. Phys. B 559 (1999) 27 [hep-ph/9904378] [INSPIRE].

[168] J.L. Feng, T. Moroi, L. Randall, M. Strassler and S.-f. Su, Discovering supersymmetry at the Tevatron in wino LSP scenarios, Phys. Rev. Lett. 83 (1999) 1731 [hep-ph/9904250] [INSPIRE].

[169] R. Schöfbeck and H. Eberl, Two-loop SUSY QCD corrections to the chargino masses in the MSSM, Eur. Phys. J. C 53 (2008) 621 [arXiv:0706.0781] [InSPIRE].

[170] R. Schöfbeck and H. Eberl, Two-loop SUSY QCD corrections to the neutralino masses in the MSSM, Phys. Lett. B 649 (2007) 67 [hep-ph/0612276] [INSPIRE].

[171] D. Pierce and A. Papadopoulos, Radiative corrections to neutralino and chargino masses in the minimal supersymmetric model, Phys. Rev. D 50 (1994) 565 [hep-ph/9312248] [INSPIRE].

[172] A.B. Lahanas, K. Tamvakis and N.D. Tracas, One loop corrections to the neutralino sector and radiative electroweak breaking in the MSSM, Phys. Lett. B 324 (1994) 387 [hep-ph/9312251] [INSPIRE].

[173] D. Pierce and A. Papadopoulos, The Complete radiative corrections to the gaugino and Higgsino masses in the minimal supersymmetric model, Nucl. Phys. B 430 (1994) 278 [hep-ph/9403240] [INSPIRE].

[174] N. Sakai, Naturalness in Supersymmetric GUTs, Zeit. Phys. C 11 (1981) 153.

[175] R.K. Kaul and P. Majumdar, Cancellation of Quadratically Divergent Mass Corrections in Globally Supersymmetric Spontaneously Broken Gauge Theories, Nucl. Phys. B 199 (1982) 36 [INSPIRE].

[176] R. Barbieri and G.F. Giudice, Upper Bounds on Supersymmetric Particle Masses, Nucl. Phys. B 306 (1988) 63 [INSPIRE].

[177] ATLAS collaboration, Further searches for squarks and gluinos in final states with jets and missing transverse momentum at $\sqrt{s}=13 \mathrm{TeV}$ with the ATLAS detector,

ATLAS-CONF-2016-078 (2016).

[178] ATLAS collaboration, Search for charginos nearly mass degenerate with the lightest neutralino based on a disappearing-track signature in pp collisions at $\sqrt{s}=8 \mathrm{TeV}$ with the ATLAS detector, Phys. Rev. D 88 (2013) 112006 [arXiv:1310.3675] [INSPIRE].

[179] CMS collaboration, Search for disappearing tracks in proton-proton collisions at $\sqrt{s}=8$ TeV, JHEP 01 (2015) 096 [arXiv: 1411.6006] [INSPIRE].

[180] ATLAS collaboration, Searches for heavy long-lived charged particles with the ATLAS detector in proton-proton collisions at $\sqrt{s}=8 \mathrm{TeV}$, JHEP 01 (2015) 068 [arXiv: 1411.6795] [INSPIRE].

[181] CMS collaboration, Search for Decays of Stopped Long-Lived Particles Produced in Proton-Proton Collisions at $\sqrt{s}=8$ TeV, Eur. Phys. J. C 75 (2015) 151 [arXiv: 1501.05603] [INSPIRE].

[182] M. Drees and M. Nojiri, Neutralino - nucleon scattering revisited, Phys. Rev. D 48 (1993) 3483 [hep-ph/9307208] [INSPIRE]. 
[183] U. Chattopadhyay, D. Das, D.K. Ghosh and M. Maity, Probing the light Higgs pole resonance annihilation of dark matter in the light of XENON100 and CDMS-II observations, Phys. Rev. D 82 (2010) 075013 [arXiv:1006.3045] [INSPIRE].

[184] XENON collaboration, E. Aprile et al., Physics reach of the XENON1T dark matter experiment, JCAP 04 (2016) 027 [arXiv: 1512.07501] [INSPIRE].

[185] B. Murakami and J.D. Wells, Nucleon scattering with higgsino and wino cold dark matter, Phys. Rev. D 64 (2001) 015001 [hep-ph/0011082] [INSPIRE].

[186] M. Low and L.-T. Wang, Neutralino dark matter at $14 \mathrm{TeV}$ and $100 \mathrm{TeV}$, JHEP 08 (2014) 161 [arXiv: 1404.0682] [INSPIRE].

[187] R. Mahbubani, P. Schwaller and J. Zurita, Closing the window for compressed Dark Sectors with disappearing charged tracks, JHEP 06 (2017) 119 [arXiv: 1703.05327] [INSPIRE].

[188] H. Fukuda, N. Nagata, H. Otono and S. Shirai, Higgsino Dark Matter or Not: Role of Disappearing Track Searches at the LHC and Future Colliders, arXiv:1703.09675 [INSPIRE]. 